\title{
Genomic insights into virulence mechanisms of Leishmania donovani: evidence from an atypical strain
}

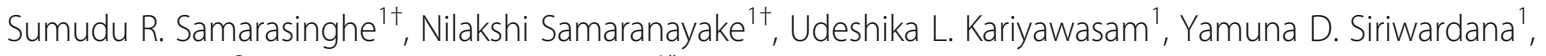
Hideo Imamura $^{2}$ and Nadira D. Karunaweera ${ }^{1^{*}}$

\begin{abstract}
Background: Leishmaniasis is a neglected tropical disease with diverse clinical phenotypes, determined by parasite, host and vector interactions. Despite the advances in molecular biology and the availability of more Leishmania genome references in recent years, the association between parasite species and distinct clinical phenotypes remains poorly understood. We present a genomic comparison of an atypical variant of Leishmania donovani from a South Asian focus, where it mostly causes cutaneous form of leishmaniasis.

Results: Clinical isolates from six cutaneous leishmaniasis patients (CL-SL); 2 of whom were poor responders to antimony (CL-PR), and two visceral leishmaniasis patients (VL-SL) were sequenced on an Illumina MiSeq platform. Chromosome aneuploidy was observed in both groups but was more frequent in CL-SL. 248 genes differed by 2 fold or more in copy number among the two groups. Genes involved in amino acid use (LdBPK_271940) and energy metabolism (LdBPK_271950), predominated the VL-SL group with the same distribution pattern reflected in gene tandem arrays. Genes encoding amastins were present in higher copy numbers in VL-SL and CL-PR as well as being among predicted pseudogenes in CL-SL. Both chromosome and SNP profiles showed CL-SL and VL-SL to form two distinct groups. While expected heterozygosity was much higher in VL-SL, SNP allele frequency patterns did not suggest potential recent recombination breakpoints. The SNP/indel profile obtained using the more recently generated PacBio sequence did not vary markedly from that based on the standard LdBPK282A1 reference. Several genes previously associated with resistance to antimonials were observed in higher copy numbers in the analysis of CL-PR. H-locus amplification was seen in one cutaneous isolate which however did not belong to the CL-PR group.

Conclusions: The data presented suggests that intra species variations at chromosome and gene level are more likely to influence differences in tropism as well as response to treatment, and contributes to greater understanding of parasite molecular mechanisms underpinning these differences. These findings should be substantiated with a larger sample number and expression/functional studies.
\end{abstract}

Keywords: Cutaneous leishmaniasis, High throughput sequencing, Aneuploidy, Copy number variations, SNPs, Drug resistance, Tropism, Indian subcontinent, Virulence genes

\footnotetext{
* Correspondence: nadira@parasit.cmb.ac.lk

tSumudu R. Samarasinghe and Nilakshi Samaranayake contributed equally to

this work.

${ }^{1}$ Department of Parasitology, Faculty of Medicine, University of Colombo,

Colombo, Sri Lanka

Full list of author information is available at the end of the article
}

(c) The Author(s). 2018 Open Access This article is distributed under the terms of the Creative Commons Attribution 4.0 International License (http://creativecommons.org/licenses/by/4.0/), which permits unrestricted use, distribution, and reproduction in any medium, provided you give appropriate credit to the original author(s) and the source, provide a link to the Creative Commons license, and indicate if changes were made. The Creative Commons Public Domain Dedication waiver (http://creativecommons.org/publicdomain/zero/1.0/) applies to the data made available in this article, unless otherwise stated. 


\section{Background}

Leishmaniasis is a neglected tropical disease with spread across 98 countries over five continents. Constituting a spectrum of diseases ranging from fatal visceral disease to localized cutaneous disease, around 350 million of the global population is at risk of developing leishmaniasis in one of its many forms [1]. Leishmaniasis is caused by over 20 species of Leishmania parasites via sandfly bites. Moreover, the same parasite species has been known to cause different clinical phenotypes. This complexity of the disease poses many challenges to control efforts which are further compounded by variable responses to drugs, not only across continents but even within the same region. While the resulting phenotype is influenced by the interactions between the parasite, susceptible host and vector as well as the environment, a more severe degree of pathogenicity such as involvement of viscera and drug resistance are considered to be virulent features largely determined by parasite genotypes.

A key approach to explore the variability of the parasite characteristics is through the study of its genome. The genomes of Leishmania vary from $29 \mathrm{Mb}$ to $33 \mathrm{Mb}$ in size and are organized into a variable number of chromosomes (i.e., 34 or 35 in new world species and 36 in old world species) [2]. The number of protein coding genes range from 8023 to 8412 [3]. However, species-specific genes per se have not provided an adequate basis to explain the wide phenotypic variability that is observed, with only a limited number of genes being differentially distributed $[4,5]$. Trypanosomatid genomes have been shown to have a peculiar arrangement amongst eukaryotes, where genes are arranged into large polycistronic gene clusters and lack conventional transcription control factors such as RNA polymerase 11 promoters. Thus, at genomic level, other mechanisms of altering gene expression such as copy number variations of chromosomes and genes have been investigated to determine parasite genetic basis to the different attributes. Single nucleotide polymorphisms (SNPs) in genes encoding vital structural and functional proteins may represent another level of such adaptations.

The advent of high throughput sequencing technologies coupled with the availability of reference genome sequences for several Leishmania species allow detailed comparative analyses at both inter and intra species level in the quest to understand these differences. The ability to cause potentially fatal visceral disease and limited treatment options has made Leishmania donovani the focus of several studies attempting to elucidate genetic factors contributing to tropism and drug resistance of this parasite. A comparative analysis of multiple isolates of $L$. donovani from patients with visceral leishmaniasis (VL) from India and Nepal showed a low level of intra species SNP variation but extensive variation in copy number in chromosomes [6]. The same study suggested a change in copy number of genes in tandem arrays and an amplicon to be associated with drug resistance. A larger study which analyzed intra species variation of L. donovani from a similar group of patients showed a genetically distinct population of parasites frequently resistant to antimonials to have a two base-pair insertion in the aquaglyceroporin (LdAQP1) gene [7].

Sri Lanka is endemic for leishmaniasis and is faced with an unusual situation where a species which causes $\mathrm{VL}$ in many parts of the Indian sub-continent, i.e. $L$. donovani, causes localized cutaneous leishmaniasis (CL) [8]. Even though a few cases of visceral disease and mucosal localization have been reported [9], the prevalent clinical type in the island continues to be almost exclusively the cutaneous form [10]. While intra-lesional or intra-muscular sodium stibogluconate (SSG) is the accepted standard therapy in the local setting, delayed response or failure to respond to treatment is not uncommon [11].

The causative parasite of both CL and VL in Sri Lanka, Leishmania donovani MON 37, has been shown to be genetically relatively close to $L$. donovani isolates from India, Bangladesh, and Nepal by both microsatellite and minicircle DNA analysis [12, 13]. A recent study comparing one isolate each from a CL and a VL patient suggested that SNPs and variable gene expression of $\operatorname{RagC}$ and $A 2$ genes are likely to be responsible for disease tropism [1]. Using next generation sequencing technologies, we describe here the genomic diversity of 8 clinical isolates of $L$. donovani from Sri Lanka; which included 2 isolates from patients diagnosed with VL and 6 isolates from patients diagnosed with CL. Among the $6 \mathrm{CL}$ patients, 2 responded poorly to antimony treatment.

\section{Results}

The six cutaneous leishmaniasis (CL-SL) and two visceral leishmaniasis (VL-SL) clinical isolates were from patients originating from different regions of the country (Additional file 1: Table S1-(A)). Raw sequencing read statistics for the parasite genomes are available in the Additional file 1: Table S1-(B).

\section{Genomic diversity in $L$. donovani isolates from $\mathrm{CL}$ and $\mathrm{VL}$ patients}

\section{Chromosome copy number/somy variations}

We used normalized read depth data to determine copy number variations in chromosomes and protein-coding genes as described in Methods (Additional file 1: Table S2). Somy variations were seen in several chromosomes among the isolates (Additional file 1: Table S3). In CL-SL isolates the majority of chromosomes were 
disomic. Deviation from this was observed in chromosome 2 with monosomy, chromosome 22 and 26 with trisomy, and chromosome 31 with pentasomy (Fig. 1a). Overall, the chromosomes were disomic in VL-SL except for chromosome 31 which was tetrasomic (Fig. 1b). Furthermore, read depth changes suggestive of possible amplifications, affecting particular sub-regions of chromosomes (for example, chr2, chr8, chr12, chr22, chr33 and chr34; Additional file 2: Figure S1 and Additional file 3: Figure S2) were also observed in our isolates.

The heat map demonstrating the aneuploidy profiles of Leishmania donovani CL-SL and VL-SL isolates further confirmed the chromosome somy variations described above (Fig. 2). As indicated in the dendrogram, based on the similarity of aneuploidy profiles, VL-SL and CL-SL isolates clustered into separate groups. Three CL-SL isolates (L2339_S2, L2343_S3 and L2372_S4), showed high similarity to each other. CL-SL poor responders to SSG (R5_S5 and R7_S6) clustered with each other but separate from the other three CL-SL isolates mentioned earlier whereas L2331_S1, the remaining
CL-SL isolate, was an exception and appeared to be more similar to the CL-SL poor drug responder group.

\section{Gene copy number and tandem arrays}

We identified 248 protein-coding genes in total with at least a 2-fold change in copy number between CL-SL and VL-SL (Additional file 1: Table S4). Genes with most varying copy numbers between $L$. donovani $\mathrm{CL}$ and $\mathrm{VL}$ isolates are summarized in Table 1.

We also identified 274 multicopy genes (tandem gene arrays) with at least a 2-fold change in gene copy number between Sri Lankan L. donovani CL and VL isolates (Additional file 1: Table S5). Multicopy genes are defined as genes of more than one copy with the same OrthoMCL group ID which are present on the same chromosome. 194 and 241 tandem gene arrays were observed in CL-SL and VL-SL respectively (Additional file 1: Table S6 and Table S7). Conforming to differences observed at individual gene level, the set of protein-coding genes with highly variable copy numbers were seen to be also present in the tandem gene arrays

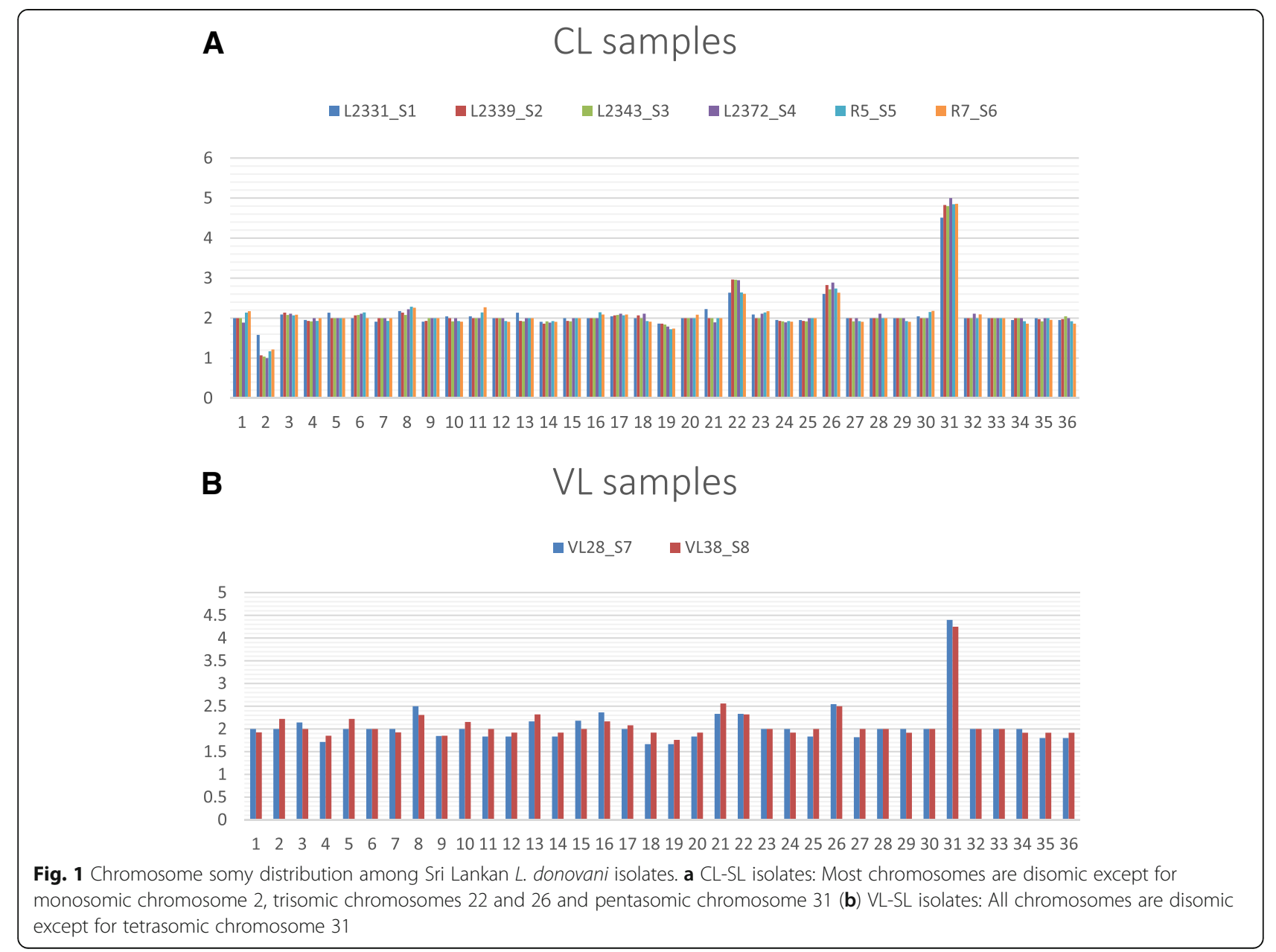




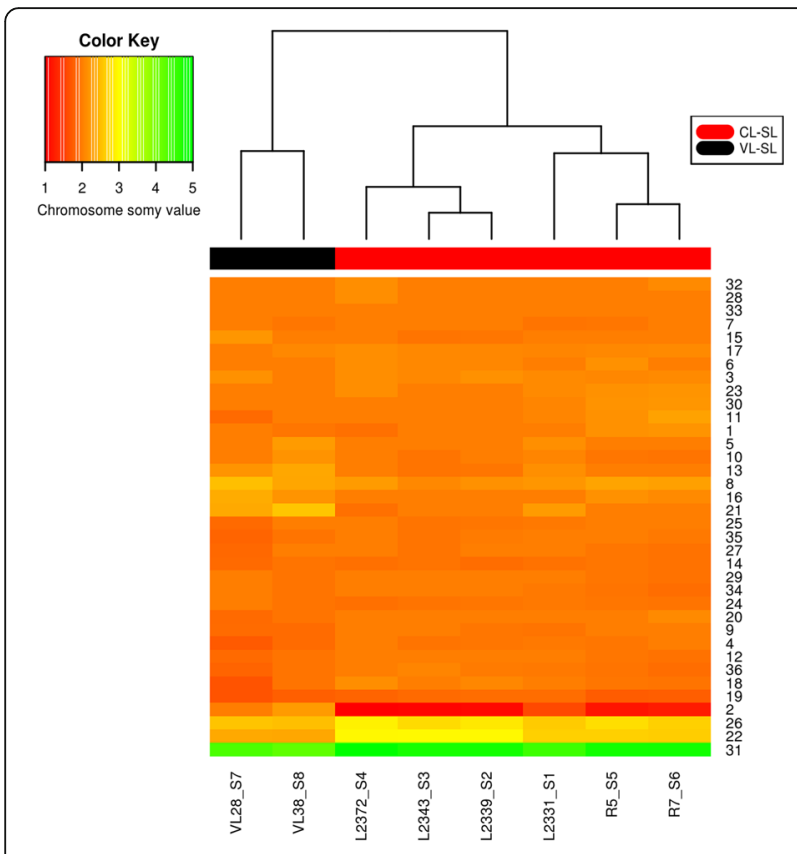

Fig. 2 Heat map showing the comparison of aneuploidy profiles of $\mathrm{CL}-\mathrm{SL}$ and $\mathrm{VL}-\mathrm{SL}$ isolates. Chromosome numbers are listed along the right side $y$-axis and $L$. donovani $C L$ and $V L$ isolates are listed along the bottom $x$-axis. The dendrogram on top of heat map indicates the clustering pattern of the 8 isolates based on their aneuploidy similarity. The colour bar below the dendrogram represents the phenotype of isolate (Red-CL-SL and Black-VL-SL). The colour key represents the ploidy level of chromosomes in the heat map: chromosome somy $>4$ green, trisomy - yellow, disomy - orange and monosomy - red

with highly variable copy numbers between CL and VL isolates (Table 2).

\section{Functional characterization of differentially distributed genes and arrays}

D-lactate dehydrogenase-like protein (LdBPK_271940, OG5_127093) and branched-chain amino acid aminotransferase (LdBPK_271950, OG5_126731) were present in significantly higher copy numbers in VL-SL group (Tables 2 and 3). D-lactate dehydrogenase-like protein is involved in energy metabolism of Leishmania and may relate to optimal growth and survival of parasites within the host. Unlike other Trypanosoma species, D-lactate dehydrogenase is present only in Leishmania and absence of this enzyme in humans has suggested this to be a candidate drug target in these parasites $[15,16]$. The extensive copy number variation seen in the branched-chain amino acid aminotransferase gene may result in variable consumption of amino acids by parasites.

Other genes with higher copy numbers in VL-SL, included GP63 gene which encodes a surface protein previously reported as an important virulence factor in Leishmania, favoring its rapid migration, internalization and survival in host macrophages $[17,18]$ whereas checkpoint protein Hus1 in Leishmania has been shown to help the parasite to cope with replicative stress [19]. Pteridine reductase 1 encoding gene is a component of the pteridine metabolic pathways, which are highly adapted among these parasites which are pteridine auxotrophs. Changes that disrupt this pathway have been found to lead to attenuation or loss of virulence, inhibiting parasite survival and growth in animal models [20-22].

Several histone encoding genes were also present in a higher copy number in VL-SL isolates. Histones form the core of the parasite nucleosome and these structural proteins play an important role in DNA metabolism including transcription regulation, DNA repair and replication. These have also been identified as evolutionarily conserved proteins which act as prominent immunogens during Leishmania infections [23]. Distinct differences in protein biosynthesis and metabolism were suggested by markedly variable copy numbers of genes such as tryptophanyl-trna synthetase (LdBPK_230340, OG5_127096) which is also identified as important drug targets in eukaryotic parasites [24] and C3HC4 type zinc-finger (LdBPK_230320, OG5_180874) which has been shown to play a key role in the ubiquitination pathway modulating protein levels [25]. Since the $A 2$ gene locus on chromosome 22 is recognized to have misassembles in the $L$. donovani BPK282A1 reference [26], we analyzed the region using the more complete $L$. donovani PacBio sequence (Additional file 4: Figure S3) but did not observe a difference in copy number among CL-SL and VL-SL. Selected genes on chromosome 27 which demonstrated marked variability in copy number among the isolates are shown in Fig. 3.

\section{GO enrichment analysis}

The genes that were differentially distributed between CL-SL and VL-SL isolates with at least a two-fold change in copy number (Additional file 1: Table S4) were analyzed for enrichment of GO terms (Additional file 1: Table S8 and Table S9). Genes identified with a higher copy number among the VL-SL group were associated with biological processes such as cell adhesion, biological adhesion, modulation by symbiont of host signal transduction pathway and macromolecular complex subunit organization, with these groups forming clear clusters (Fig. 4a). This is a likely reflection of the role of these processes in conferring parasite virulence features. The high copy number genes within the CL-SL group were associated with biological processes related to cellular response to oxidative stress, response to abiotic stimulus, response to chemicals and response to heat and proteolysis (Fig. 4b), indicating the cutaneous parasite's adaptation to changes in the environment. Differences were also noted in the molecular functions of the two groups. High copy number genes in VL-SL were mainly 
Table 1 Genes with highly variable copy numbers between Sri Lankan L. donovani CL-SL and VL-SL isolates

\begin{tabular}{|c|c|c|c|c|c|}
\hline Chr & GenelD & $\begin{array}{l}\mathrm{VL} / \mathrm{CL} \\
\text { Ratio }\end{array}$ & $\begin{array}{l}\text { MWU-test p- } \\
\text { value }\end{array}$ & $\begin{array}{l}\text { Benjamini-Hochberg p- } \\
\text { value }\end{array}$ & Product Description \\
\hline 27 & LdBPK_271940 & 7.96 & 0.2717 & 0.3702 & D-Lactate Dehydrogenase-Like Protein \\
\hline 27 & LdBPK_271950 & 6.84 & 0.0012 & 0.0029 & $\begin{array}{l}\text { Branched-Chain Amino Acid Aminotransferase, Putative } \\
\text { (Fragment) }\end{array}$ \\
\hline 10 & LdBPK_100520 & 4.698 & 0.0000 & 0.0000 & Gp63, Leishmanolysin \\
\hline 29 & LdBPK_291600 & 4.41 & 0.0000 & 0.0000 & Nodulin-Like, Putative \\
\hline 23 & LdBPK_230360 & 4.22 & 0.0000 & 0.0000 & Hypothetical Protein, Conserved \\
\hline 23 & LdBPK_230350 & 3.86 & 0.0022 & 0.0050 & Hypothetical Protein, Conserved \\
\hline 23 & LdBPK_230330 & 3.85 & 0.0133 & 0.0264 & Checkpoint Protein Hus1, Putative \\
\hline 25 & LdBPK_210020 & 3.80 & 0.0009 & 0.0023 & Histone H4 \\
\hline 10 & LdBPK_100920 & 3.55 & 0.8462 & 0.8817 & Histone H3 \\
\hline 23 & LdBPK_230340 & 3.47 & 0.0001 & 0.0004 & Tryptophanyl-Trna Synthetase, Putative \\
\hline 23 & LdBPK_230320 & 3.06 & 0.2234 & 0.3163 & Zinc Finger, C3Hc4 Type (Ring Finger), Putative \\
\hline 23 & LdBPK_230310 & 3.02 & 0.7210 & 0.7877 & Pteridine Reductase 1 \\
\hline 11 & LdBPK_110640 & 2.95 & 0.0922 & 0.1456 & Metallo-Peptidase, Clan Mf, Family M17 (Fragment) \\
\hline 29 & LdBPK_291590 & 2.90 & 0.3598 & 0.4576 & Asparagine Synthase, Putative \\
\hline 10 & LdBPK_100510 & 2.55 & 0.2285 & 0.3201 & Gp63, Leishmanolysin (Fragment) \\
\hline 23 & LdBPK_230290* & 2.49 & 0.8027 & 0.8474 & Abc-Thiol Transporter (Fragment) \\
\hline 23 & LdBPK_230300 & 2.47 & 0.8735 & 0.9026 & Argininosuccinate Synthase, Putative \\
\hline 23 & LdBPK_230250* & 2.47 & 0.0540 & 0.0905 & Hypothetical Protein, Conserved \\
\hline 35 & LdBPK_353690 & 2.38 & 0.0001 & 0.0003 & Hypothetical Protein, Conserved \\
\hline 22 & LdBPK_221370 & 2.36 & 0.0350 & 0.0616 & 40S Ribosomal Protein L14, Putative \\
\hline 31 & LdBPK_310470 & 2.28 & 0.6628 & 0.7405 & Hypothetical Protein \\
\hline 23 & LdBPK_230370 & 2.27 & 0.0000 & 0.0000 & Hypothetical Protein, Conserved \\
\hline 23 & LdBPK_230260* & 2.22 & 0.1946 & 0.2790 & Hypothetical Protein, Conserved \\
\hline 25 & LdBPK_020010 & 2.20 & 0.1855 & 0.2691 & Aminopeptidase P1, Putative \\
\hline 15 & LdBPK_150430 & 2.19 & 0.9484 & 0.9561 & 605 Acidic Ribosomal Protein, Putative \\
\hline 27 & LdBPK_271490 & 2.13 & 0.1280 & 0.1947 & Hypothetical Protein, Conserved \\
\hline 11 & LdBPK_111070 & 2.13 & 0.3303 & 0.4289 & Hypothetical Protein \\
\hline 28 & LdBPK_282050 & 2.12 & 0.4786 & 0.5652 & Zinc Transporter 3, Putative \\
\hline 34 & LdBPK_341720 & 2.10 & 0.9113 & 0.9300 & Amastin-Like Surface Protein, Putative (Fragment) \\
\hline
\end{tabular}

Benjamini-Hochberg critical value for a false discovery rate $<0.05$ was considered significant and marked in bold

${ }^{*}$ Genes previously reported with high copy number variations between a cutaneous and a visceral isolate from Sri Lanka [14]

associated with functions such as protein polymerization while peptidase activity was one of the main associations of the genes in the CL-SL group.

\section{SNP/Indel diversity}

A high quality set of SNPs and indels were obtained by site and genotype-level filtering of the raw SNP and indel set produced by GATK's HaplotypeCaller as described in methods. We identified 85,642 variants (81,859 SNPs and 3783 indels) consistent within the 6 clinical samples of CL-SL against $L$. donovani reference. There were 69,841 variants $(68,008$ SNPs and 1833 indels) consistent within the 2 clinical samples of VL-SL against the $L$. donovani reference. Of these 56,560 SNPs and 1674 indels were common to isolates from the two phenotypes. 21,799 SNPs and 2109 indels were unique to CL-SL while 8688 SNPs and 159 indels were unique to VL-SL.

Varying from the chromosome aneuploidy profiles, the heat map generated based on a similarity measure between the SNP profiles of 8 Sri Lankan isolates showed that they formed three distinct groups, which could also be related to their associated phenotype (Fig. 5a). Principal component analysis on the SNPs showed a similar population structure (Fig. 5b). Figure 5(c) represents the phylogenetic relationship between the 8 Sri Lankan $L$. donovani isolates and other key Leishmania strains described in a previous study [7]. Overall topology of the 
Table 2 Tandem gene arrays with highly variable copy numbers between L. donovani CL-SL and VL-SL isolates

\begin{tabular}{|c|c|c|c|c|c|c|}
\hline Chr & $\begin{array}{l}\text { OrthoMCL } \\
\text { ID }\end{array}$ & Product description & $\begin{array}{l}\text { No of genes in } \\
\text { ref }\end{array}$ & $\begin{array}{l}\text { Haploid number } \\
\mathrm{CL}\end{array}$ & $\begin{array}{l}\text { Haploid number } \\
\text { VL }\end{array}$ & $\begin{array}{l}\mathrm{VL} / \mathrm{CL} \\
\text { ratio }\end{array}$ \\
\hline 27 & OG5_127093 & D-lactate dehydrogenase-like protein & 1 & 4 & 33 & 7.96 \\
\hline 27 & OG5_126731 & Branched-chain amino acid aminotransferase, putative & 1 & 5 & 35 & 6.84 \\
\hline 29 & OG5_131290 & Nodulin-like, putative & 1 & 1 & 6 & 4.41 \\
\hline 23 & OG5_154577 & Hypothetical protein, conserved & 1 & 1 & 4 & 4.22 \\
\hline 23 & OG5_181194 & Hypothetical protein, conserved & 1 & 1 & 5 & 3.86 \\
\hline 23 & OG5_150800 & Checkpoint protein Hus1 & 1 & 1 & 5 & 3.85 \\
\hline 21 & OG5_126575 & Histone $\mathrm{H} 4$ & 1 & 1 & 2 & 3.80 \\
\hline 23 & OG5_127096 & Tryptophanyl-trna synthetase, putative & 1 & 1 & 5 & 3.47 \\
\hline 23 & OG5_180874 & $\begin{array}{l}\text { Zinc finger, } \mathrm{C} 3 \mathrm{H} c 4 \text { type (ring finger) containing protein, } \\
\text { putative }\end{array}$ & 1 & 1 & 4 & 3.06 \\
\hline 23 & OG5_133937 & Pteridine reductase 1 & 1 & 1 & 4 & 3.02 \\
\hline 10 & OG5_126749 & Major surface protease Gp63, putative & 2 & 7 & 21 & 3.00 \\
\hline 34 & OG5_130729 & Amastin-like surface protein-like protein & 2 & 4 & 2 & 0.50 \\
\hline 22 & OG5_126823 & $\begin{array}{l}\text { Ribonucleoside-diphosphate reductase small chain, } \\
\text { putative }\end{array}$ & 3 & 2 & 1 & 0.50 \\
\hline 12 & OG5_136657 & Protein of unknown function (Duf962), putative & 2 & 2 & 1 & 0.50 \\
\hline 8 & OG5_132981 & Amastin, putative & 1 & 2 & 1 & 0.44 \\
\hline
\end{tabular}

tree for the samples previously described by Imamura $\mathrm{H}$ and colleagues is similar to our current tree. This tree represents the genetic diversity of $L$. donovani in Sri Lanka that we examined, and is supported by many definitive homozygous SNPs. Although we still observed some base noises due to mismatched bases in the alignment, we have eliminated most of the potential computational errors such as missing information and low quality SNPs.

\section{Functional variation of SNPs in protein-coding regions}

Among the variants unique to CL-SL, 3169 SNPs were present in 1611 coding DNA sequences (CDS) and 34 indels in 27 CDS (Additional file 1: Table S10 and S11). Similarly, among the variants unique to VL-SL, we found 5357 SNPs in 1686 CDS and 54 indels in 43 CDS
(Additional file 1: Table S12 and S13). The SNPs and their functional effects are summarized in Table 3.

Our results showed that 17 genes in CL-SL and 32 genes in VL-SL have been affected by frame shifts or stop codon variations unique to the isolates that could possibly give rise to pseudogenes and affect protein expression (Additional file 1: Table S14). Among the predicted pseudogenes in CL-SL isolates, some genes were identified with important putative functions. Amastin like surface proteins (LdBPK_044340) are abundant surface antigens which are known to be highly expressed in amastigote stage of Leishmania parasites. These are vital transporters in parasite survival inside host cells and are also signal transducers that allow the parasite to adapt to host environment [27]. Trypanothione reductase (LdBPK_050350), is a critically important enzyme for

Table 3 Effects of SNPs and short indels identified in Sri Lankan L. donovani isolates on coding regions

\begin{tabular}{llll}
\hline Variant type & Common to $V L \& C L$ & Unique to $C L$ & Unique to $V L$ \\
\hline Frame shift & 52 & 15 & 31 \\
Stop gained & 21 & 3 & 7 \\
Stop lost & 8 & 2 & 4 \\
Start lost & 2 & 2 & 3 \\
Stop retained & 13 & 3 & 2804 \\
Non synonymous coding & 12,046 & 1576 & 2507 \\
Synonymous coding & 12,181 & 1558 & - \\
Initiator codon variant & 1 & - & 3 \\
Disruptive inframe deletion & 30 & 4 & 10 \\
Disruptive inframe insertion & 35 & 4 & \\
\hline
\end{tabular}



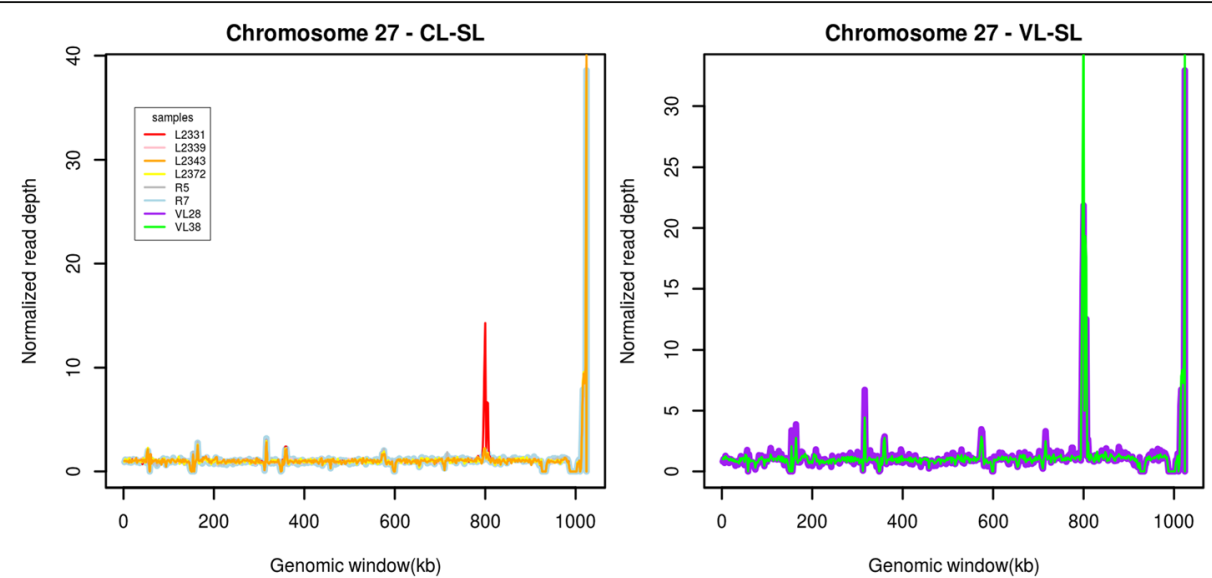

Fig. 3 Genes with highly variable copy numbers on chromosome 27 among CL-SL and VL-SL isolates. LdBPK_271940 (Chr27: 798359-799,840) and LdBPK_271950 (Chr27: 803591-804,793). The two genes show a drastic increase in copy number among VL-SL isolates (VL28 and VL38) compared to CL-SL isolates (L2339, L2343, L2372, R5 and R7). As an exception L2331 CL isolate (red) shows a somewhat higher increase in copy number of the genes than other CL-SL isolates. Figure was generated using R graphics [73]

Leishmania parasite survival and their ability to survive inside macrophages can be significantly reduced due to partial loss of this protein activity [28] (Additional file 5: Figure S4). Similarly, the possible pseudogenes predicted among the VL-SL isolates include functionally important genes as well. Putative mitogen-activated protein kinases/MAPKs (LdBPK_020260, LdBPK_131380) are found to be vital components in Leishmania signaling cascades. Past studies have reported that inhibition of MAPKs activity relates to the increased survival of $L$. donovani parasites in human peripheral blood mononuclear macrophages [29].

Among the high SNP count genes in CL-SL and VL-SL, 685 and 734 genes respectively (Additional file 1 : Table S15 and S16) were identified as genes under strong selection using SNP ratios as described in methods, possibly indicating the importance of these gene functions in Leishmania biology.

The expected heterozygosity $(\mathrm{He})$ which is a measure for genetic diversity was higher in VL-SL isolates, with a negative inbreeding coefficient (Table 4). L2231 CL-SL isolate was more similar to VL-SL group with a higher number of heterozygous SNPs and a negative inbreeding coefficient. Among the other CL-SL isolates, the two isolates from poor responders to sodium stibogluconate (R5 and R7) appeared to be similar to each other. There were no distinct changes in allele profiles that were demonstrated by changes in heterozygous SNP coverage depth, and this indicated that there were no clear signs of potential recent recombination breakpoints on any of the chromosomes in the eight isolates even though losses of heterozygosity, where heterozygous SNPs were lost in a large block, were observed (Additional file 6: Figure S5).
SNP/Indel analysis using a new genomic sequence of $L$. donovani

Refining existing Leishmania references using a PacBio sequencing platform have been reported in several Leishmania species, which improved both coverage and base accuracy of the repetitive regions of the current genome references [30, 31]. We used a more recently generated $L$. donovani genome sequence to complement our findings. The same protocol as described under methods was used to call variants. The results indicated that the SNP/indel counts generated using the more recent PacBio sequence and the standard LdBPK282A1 reference are compatible and did not vary markedly, partly because SNPs on repetitive regions with lower mapping quality were screened out (Table 5). A marginal increase in the total number of variants generated with the PacBio sequence is in agreement with the fact that it became slightly longer and has a lesser number of misassembles when compared to the standard LdBPK282A1 reference.

\section{Poor response to SSG among cutaneous isolates of $L$. donovani \\ Chromosome and gene copy number variations in comparison to drug response}

Chromosome copy numbers were not remarkably variable among the CL poor responders to SSG (CL-PR) and CL wild type (CL-WT) (Fig. 1a, Additional file 1: Table S3) in agreement with an earlier study that explains antimonial resistance in $L$. donovani clinical isolates [6]. Coverage analysis showed that $\mathrm{H}$-locus region on chromosome 23 (Fig. 6), which includes ATP-binding cassette ABC transporter gene MRPA (LdBPK_230290), had notably amplified in our VL isolates. Among the six $\mathrm{CL}$ isolates this 


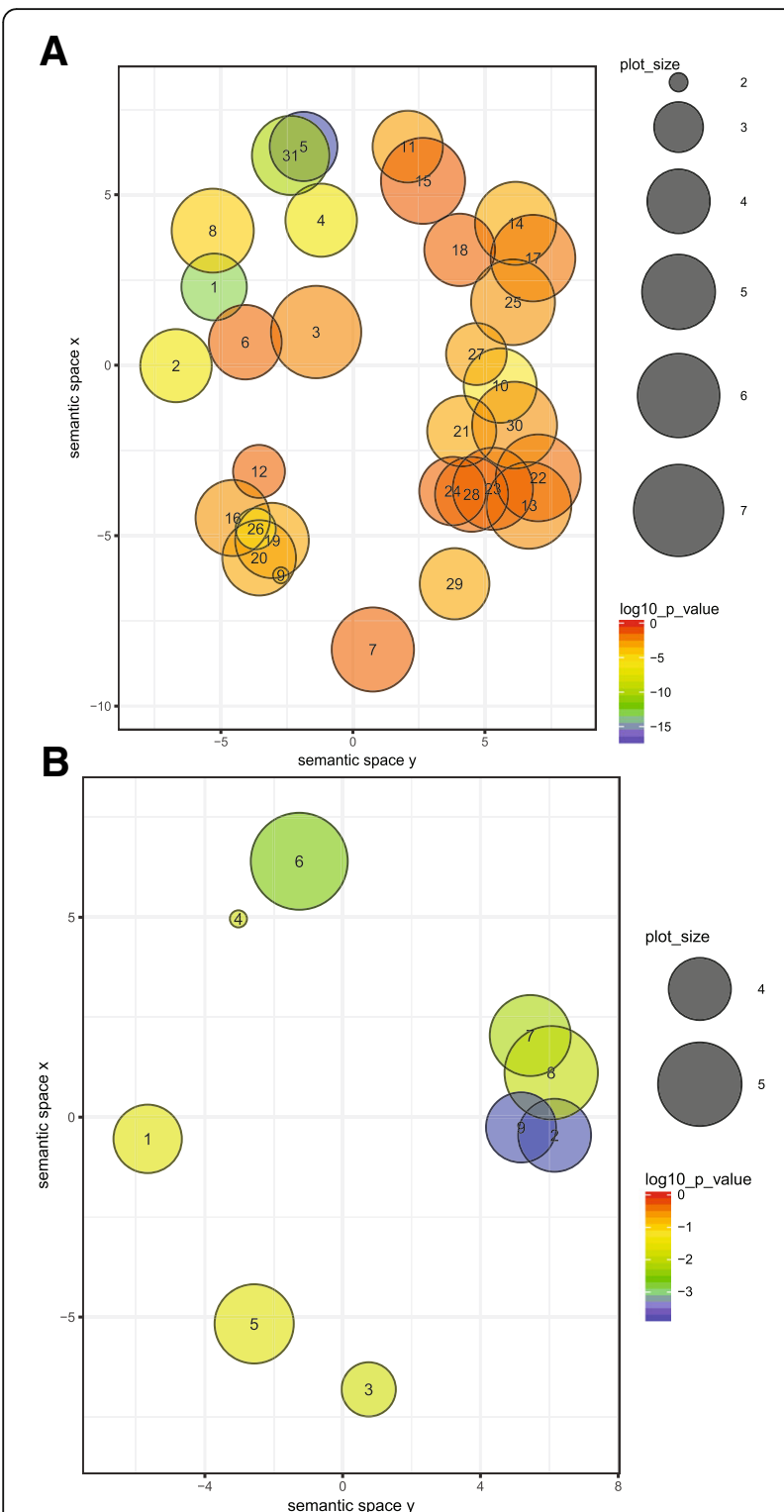

Fig. 4 Enriched GO terms within the highly variable genes among $\mathrm{CL}-\mathrm{SL}$ and $\mathrm{VL}-\mathrm{SL}$ isolates. GO terms found to be overrepresented with a $p<0.05$ are shown for biological processes in VL-SL (Panel a) and CL-SL (Panel b). A web-based tool, REVIGO was used to remove redundant $\mathrm{GO}$ terms among the gene set based on a chosen semantic similarity measure of 0.5 . Remaining $\mathrm{GO}$ terms were represented as circles in a two dimensional scatter plot, where the size of the circles indicates the frequency of the GO term it represents and the distance between the circles indicates the semantic similarity between the corresponding $\mathrm{GO}$ terms. Circles closer to each other in the plot represent semantically similar GO terms. Circle color indicates the enriched GO term $p$-values according to the color key given in the plot. Number within each circle corresponds to the GO term as per Additional file 1: Table S9. Plot axes $x$ and $y$ is a general representative measure of semantic space amplification was seen in only one isolate which however did not show a poor response to intra-lesional stibogluconate. Interestingly, the two $\mathrm{CL}$ isolates associated with a poor response to SSG in this study (R5 and R7) did not show a notable increase at the H-locus. The sensitivity of the two local VL isolates to stibogluconate is not known as the patients were treated with liposomal Amphotericin B as per local guidelines.

In addition to $M R P A, \mathrm{H}$-locus comprises of another two important genes, terbinafine resistance locus protein (LdBPK_230280) and argininosuccinate synthase gene (LdBPK_230300). Terbinafine resistance locus protein has previously been identified to mediate efflux of terbinafine in L. major [32] and argininosuccinate synthase gene has been identified to be associated with antimony resistance in L. infantum [33]. Also an up regulation of argininosuccinate synthase gene was seen in Leishmania parasites as a response to oxidative stress and arginine starvation and the viability of the parasites were seen to notably reduce when the gene was down regulated, indicating an important role of the argininosuccinate synthase gene in infectivity and survival of the parasites in the mammalian host $[34,35]$.

We identified a total of 52 genes with at least a 2 -fold change in gene copy number between CL-WT and CL-PR to SSG (Additional file 1: Table S17). However, only 27 genes showed a statistically significant change (FDR $<0.05$ with Benjamini-Hochberg correction) between the two groups, where most of them were hypothetical proteins (Table 6). We identified an important gene encoding tryparedoxin peroxidase (LdBPK_151140) and also Cathepsin L-like protease with a relatively higher copy number in the CL-PR group, which was previously shown to have crucial roles related to antimony resistance in $L$. donovani and its survival as well as virulence [36]. Cysteine proteases (Cathepsin L- and B-like cysteine proteases) have been found to play an important role in pathogenesis and survival within the host macrophages in Leishmania and also have been identified as targets for drug design [37, 38].

We identified 186 tandem arrays in CL-WT (Additional file 1: Table S18) while there were 191 tandem arrays in CL-PR group (Additional file 1: Table S19). Interestingly, only 16 arrays had at least a 2 -fold change in copy number between the two groups (Table 7, Additional file 1: Table S20). Among them quinonoid dihydropteridine reductase (QDPR) (OG5_131023), amastin surface glycoprotein (OG5_143904, LdBPK_341720.1) and histidine secretory acid phosphatase (OG5_158724) have shown an increase in its gene copy number in CL-PR group. QDPR has been characterized in Leishmania major and reported as a key enzyme required for regeneration and maintenance of $\mathrm{H}_{4}$ biopterin, a molecule found to be crucial for growth, virulence and differentiation of Leishmania parasites [39]. 


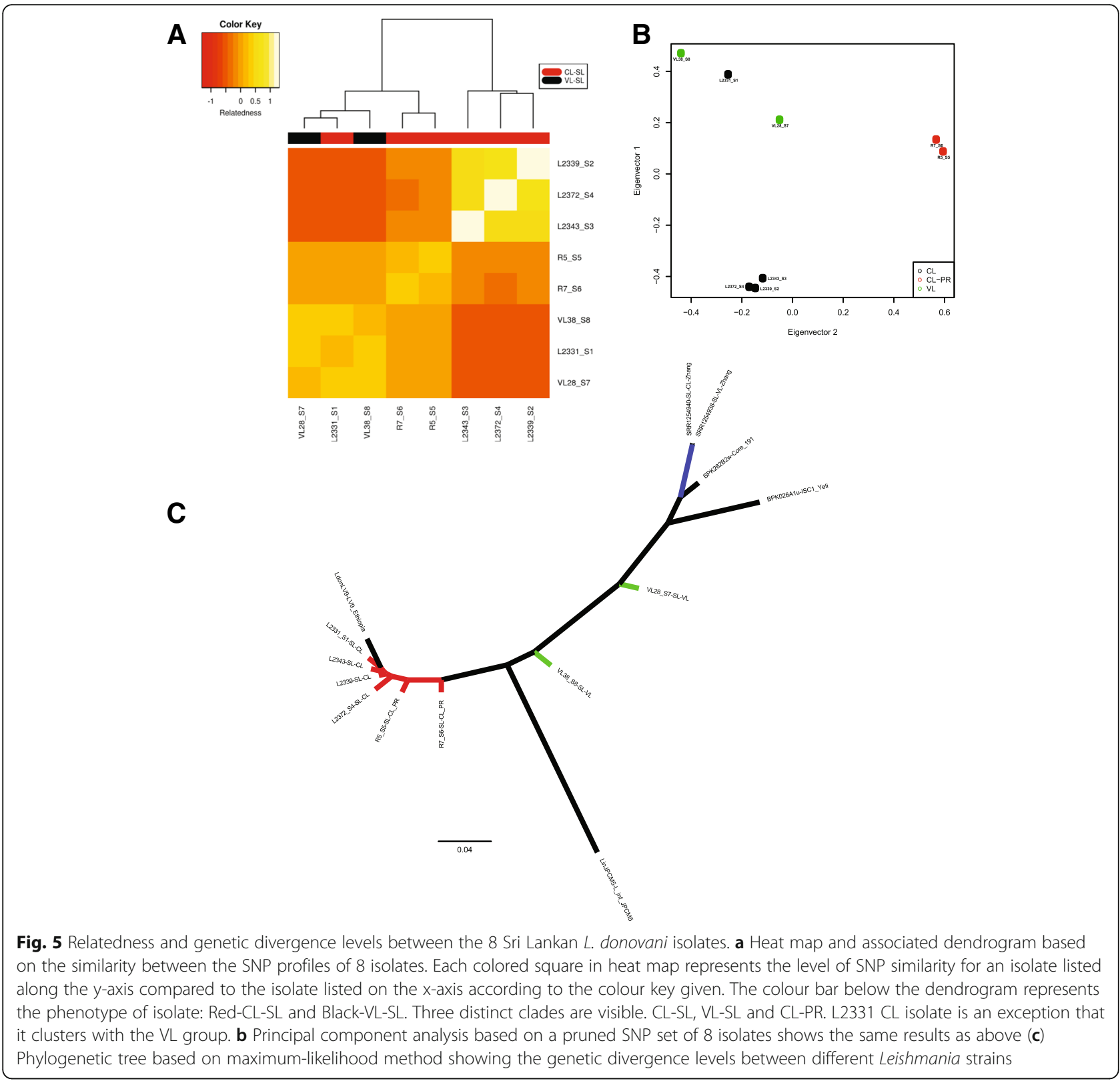

We observed a 2-fold increase in copy number of a tandem gene array encoding highly immunogenic amastin surface glycoprotein (OG5_143904, LdBPK_341720.1) in CL-PR group. Leishmania amastins are surface antigens predominantly expressed in amastigote stage of parasite's life cycle. They are identified as essential transporters and signal transducers that help the parasites to adapt to changing environment within the host $[27,40]$. A previous study has reported a gene which encodes an amastin (LdBPK_080760), with a significantly higher copy number in Leishmania parasites that have shown resistant to SSG in a clinical setting [6]. We observed the LdBPK_080760 gene within our previously discussed set of 52 genes with at least a 2-fold change in copy number (Additional file 1: Table S17). Hence we may argue that parasites use differential expression of amastins as a means of adapting to induced antimony pressure within host.

A tandem gene array (OG5_158724) consisting two gene types: LdBPK_366770 encoding a histidine secretory acid phosphatase and LdBPK_366740 encoding a tartrate-sensitive acid phosphatase showed a 2-fold increase in copy number among our poor responders to SSG (Table 7). Both these genes have been previously reported with a significantly higher copy number in a set of SSG resistant clinical isolates [6]. 
Table 4 Heterozygosity and homozygosity within the CL-SL and VL-SL genomes

\begin{tabular}{lllll}
\hline Isolate & $H_{\circ}$ & $H_{e}$ & Total SNPs & $F$ (inbreeding coefficient) \\
\hline VL38 & 26,663 & 57,592 & 84,255 & -1.28637 \\
L2331 & 38,248 & 58,153 & 96,401 & -1.21113 \\
VL28 & 13,098 & 24,524 & 47,622 & -1.10192 \\
R7 & 39,283 & 41,672 & 80,955 & -0.77418 \\
R5 & 44,411 & 40,095 & 84,506 & -0.65821 \\
L2343 & 56,029 & 6051 & 62,080 & 0.74412 \\
L2339 & 57,733 & 4071 & 61,804 & 0.83690 \\
L2372 & 52,717 & 2273 & 54,990 & 0.90426
\end{tabular}

Heterozygosity and homozygosity identified within the 8 isolate genomes, among a total of 100,318 SNPs. Number of heterozygous SNPs $\left(H_{e}\right)_{\text {, }}$ homozygous SNPs $\left(\mathrm{H}_{\mathrm{o}}\right)$ and inbreeding coefficient $(\mathrm{F})$ were calculated using VCFtools

\section{SNP/Indel distribution in comparison to drug response}

We detected a total of 15,076 SNPs distributed in 3385 protein coding genes among the CL- PR group, of which 7361 were non-synonymous and 7665 were synonymous mutations. Genes annotated with high counts of non-synonymous SNPs mostly encoded hypothetical proteins. In addition, a phosphatidylinositol 3-kinase-like protein, a putative $\mathrm{ABC} 1$ transporter, putative Vps51/ Vps67 and a putative glycosyl transferase were detected (Additional file 1: Table S21). Phosphatidylinositol 3-kinase-like protein and Vps51/Vps67 genes were annotated with high counts of non-synonymous SNPs among our CL-PR group. A non-synonymous coding SNP (Chromosome 2, position 36,464) in phosphatidylinositol 3-kinase (LdBPK_020100) has previously been identified to be highly differentiated between SSG resistant and susceptible lines [6]. Vps51/Vps67 domain as well as phosphatidylinositol 3-kinase domain have been found to be associated with TOR signaling pathway in Trypanosomes [41] possibly indicating the importance of this pathway in relation to SSG poor response in Leishmania.

Variants confined to CL-PR group are candidates for further study to investigate their functional role in causing poor response to SSG. We found 100 variants which could possibly result in pseudogenes (81 frame shifts, 2 stop lost and 17 stop gained) distributed among 71 genes in the Sri Lankan CL-PR group, while only 26 variants (24 frame shifts and 2 stop lost) were identified among 16 genes in the Sri Lankan CL wild type (Additional file 1: Table S22). Interestingly, a mitogen-activated protein kinase produces a pseudogene within our poor responders, down regulation of which has recently been found to be associated with antimony resistance in Leishmania field isolates [42].

\section{Discussion}

The spectrum of clinical presentations in leishmaniasis as well as growing resistance to the limited number of effective drugs have resulted in many efforts to understand the parasite factors underlying these mechanisms. Developments in next generation sequencing technologies have facilitated in depth exploration of Leishmania parasites differing in these characteristics. This study focused on analysis and comparison of eight genomes of Leishmania donovani from Sri Lanka; isolated from six patients with CL and two patients with VL. Two of the $\mathrm{CL}$ isolates were from patients who demonstrated a poor response to antimonials.

Diversity in the genomes from the two phenotypes were suggested by our analysis based on chromosome and gene copy number variations as well as SNP distribution. Chromosome copy number/somy variations are common and well tolerated among Leishmania species $[30,43]$. While analysis of chromosome somy showed a mostly disomic pattern in both groups there were some notable exceptions. A previous study of Sri Lankan $L$. donovani [14] describes chromosome 23 to be trisomic in CL-SL and VL-SL, but we observed a disomic pattern in chromosome 23 in both groups. The same study explains a disomic pattern in chromosome 13 and 20 in CL-SL and a trisomic pattern of same in VL-SL. In contrast, we observed a disomic pattern in chromosome 13 and 20 in both CL-SL and VL-SL. Monosomy is a relatively rare phenomenon in Leishmania with a few other reports of similar findings. For instance, in the analysis of two Leishmania (Viannia) peruviana isolates, chromosomes 1 to 5 in both were seen to be closer to monosomy [44] while Chromosome 2 was reported to be predominantly monosomic in a study of Leishmania major Friedlin (LmjF) strain [45]. Variation in somy per individual chromosome within the same species has

Table 5 Comparison of SNP/Indel profiling against different sequences of $L$. donovani

\begin{tabular}{|c|c|c|c|c|}
\hline & \multicolumn{2}{|l|}{ SNP count } & \multicolumn{2}{|l|}{ Indel count } \\
\hline & $\begin{array}{l}\text { LdBPK282A1 (Standard } \\
\text { reference) }\end{array}$ & $\begin{array}{l}\text { LdBPKPAC2016beta (PacBio } \\
\text { sequence) }\end{array}$ & $\begin{array}{l}\text { LdBPK282A1 (Standard } \\
\text { reference) }\end{array}$ & $\begin{array}{l}\text { LdBPKPAC2016beta (PacBio } \\
\text { sequence) }\end{array}$ \\
\hline $\begin{array}{l}\text { Shared by both } \mathrm{CL} \\
\text { and } \mathrm{VL}\end{array}$ & 56,560 & 57,063 & 1674 & 1669 \\
\hline Unique to $\mathrm{CL}$ & 21,799 & 24,858 & 2109 & 2140 \\
\hline Unique to VL & 8688 & 7920 & 159 & 161 \\
\hline
\end{tabular}




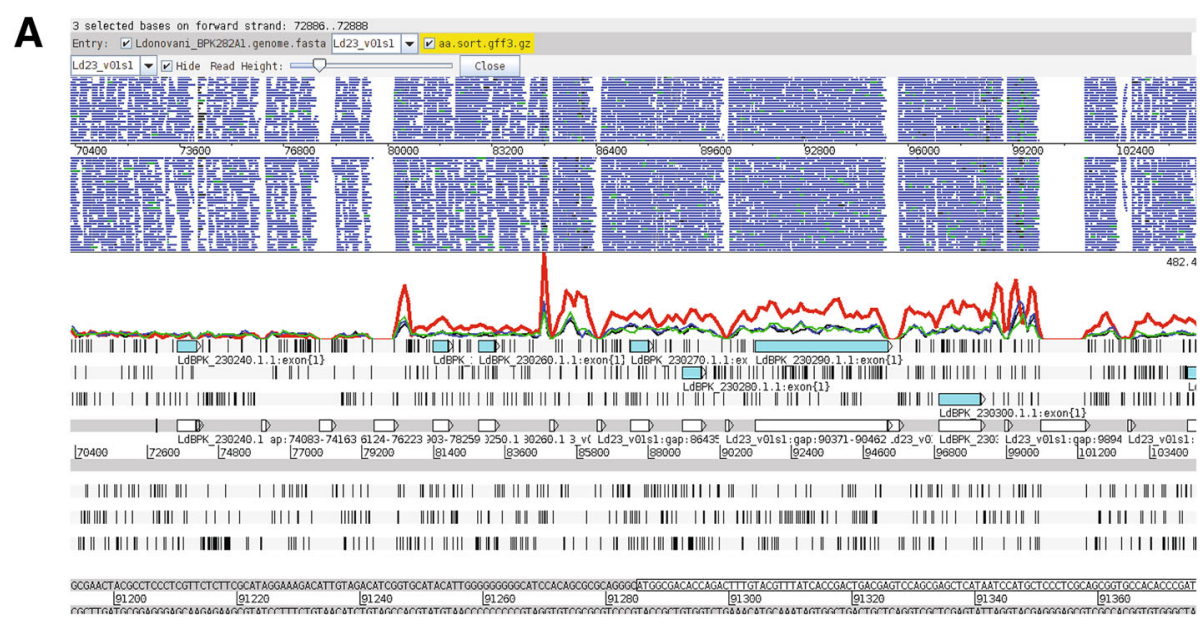

B
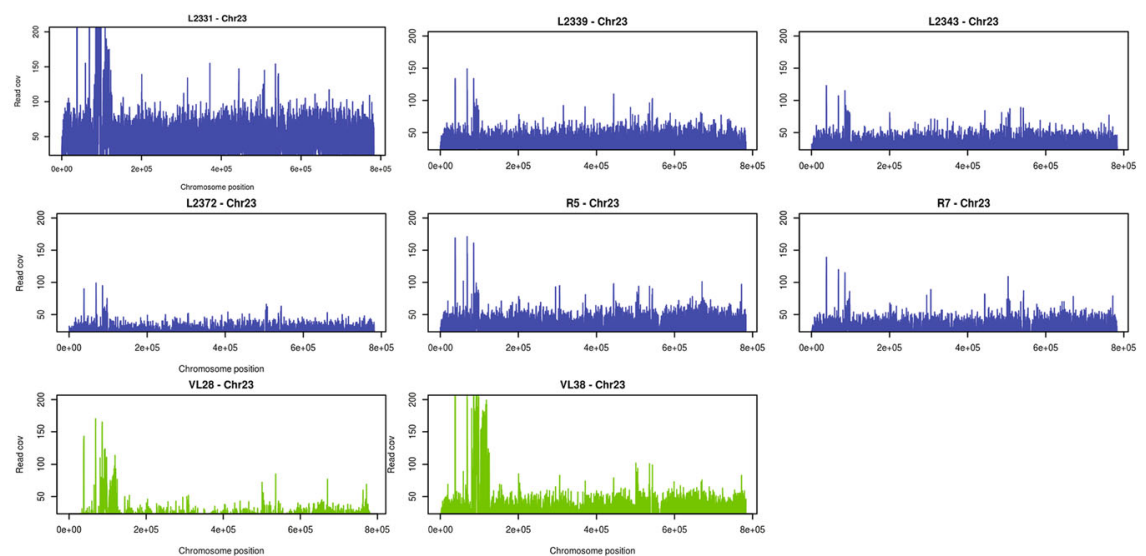

Fig. 6 Comparison of read coverage across H-locus region of chromosome 23 among Sri Lankan L. donovani CL and VL isolates. a L. donovani $\mathrm{H}$-locus region is defined between the coordinates $80,157 \mathrm{bp}-102,529 \mathrm{bp}$ on chromosome 23 . Few isolates were chosen randomly among $\mathrm{CL}$ and VL groups to clearly represent the difference in amplification of the H locus region; CL isolates - L2339 (Green), L2343 (Blue), R5 (Black) and VL isolate - VL38 (Red). Amplification of the H-locus region in VL-SL compared to CL-SL isolates were clearly observed in genes; Terbinafine resistance locus protein (LdBPK_230280: 89042-89,611), MRPA (LdBPK_230290: 91285-95,325) and Argininosuccinate synthase gene (LdBPK_230300: 96936-98,192). Figure was generated using Artemis tool (b) Read coverage across chromosome 23 in six CL-SL and two VL-SL L. donovani isolates. Amplification of the H-locus $(80,157 \mathrm{bp}-102,529 \mathrm{bp})$ is clearly seen among VL-SL VL38 isolate (In VL28 the H-locus amplification was not prominent probably due to comparatively low coverage of the sample) and one CL-SL isolate was an exception (L2331). Figure was generated using R graphics

been observed in $L$. donovani where phylogenetically close strains exhibited a different somy for 8 chromosomes including monosomy in chromosome 2 [6]. Chromosome 31 has been previously identified as supernumerary in all Leishmania species [43], generally tetrasomic in $L$. donovani [6]. Presence of aneuploidy is found to be common among Leishmania species while $L$. infantum and $L$. donovani have been previously identified to be the most aneuploid at the population level $[6,43,45]$. Aneuploidy is known to be closely mirrored at the transcript level thus affecting gene expression modulation [30, 46, 47].

We observed noninteger values for chromosome somy in several samples of CL and VL isolates. Such observations have been suggested to indicate mosaic aneuploidy; with varying levels of chromosomal content within a cell population [6,30], which could result in intra-strain differences in parasite characteristics. Overall variability in ploidy in closely related isolates, as seen in the comparison of CL-SL and VL-SL could also be due to differences in exposure to in vitro conditions such as the number of passages [30], which however is an unlikely cause in the current isolates. Regional/segmental amplifications can occur through intrachromosomal rearrangements and have been reported in different laboratory settings [48, 49], as well as in natural parasite populations [7] as a response to drug pressure or other stressful conditions.

Our results also showed that the total number of arrays and the number of gene copies within an array varies among isolates of the same species which cause different phenotypes. Such increased gene content due 
Table 6 Genes with highly variable copy numbers between L. donovani CL-WT and CL-PR

\begin{tabular}{llllll}
\hline Chr & GenelD & CL-PR/CL-WT ratio & MWU-test p-value & Benjamini-Hochberg p-value & Product Description \\
\hline 32 & LdBPK_323700 & 3.42 & 0.0032 & 0.0087 & Hypothetical Protein, Conserved \\
34 & LdBPK_044270 & 2.35 & 0.1976 & 0.2635 & Hypothetical Protein, Conserved \\
17 & LdBPK_081310 & 2.26 & 0.4180 & 0.4932 & Hypothetical Protein, Conserved \\
34 & LdBPK_341300 & 1.86 & 0.0102 & 0.0221 & Hypothetical Protein, Conserved \\
1 & LdBPK_010160 & 1.83 & 0.0167 & 0.0347 & Lsm Domain Containing Protein, Putative \\
32 & LdBPK_322060 & 1.80 & 0.0245 & 0.0471 & Hypothetical Protein \\
34 & LdBPK_341720 & 1.80 & 0.0839 & 0.1364 & Amastin-Like Surface Protein, Putative (Fragment) \\
12 & LdBPK_120667 & 1.79 & 0.0000 & 0.0000 & Hypothetical Protein (Fragment) \\
12 & LdBPK_120210 & 1.70 & 0.1487 & 0.2092 & Hypothetical Protein, Conserved \\
19 & LdBPK_191070 & 1.69 & 0.0054 & 0.0134 & Hypothetical Protein, Unknown Function \\
9 & LdBPK_090180 & 1.68 & 0.4268 & 0.4932 & Atg8/Aut7/Apg8/Paz2, Putative \\
21 & LdBPK_211640 & 1.68 & 0.0000 & 0.0001 & Zinc Finger Domain-Like Protein \\
30 & LdBPK_301560 & 1.68 & 0.3125 & 0.3964 & Hypothetical Protein \\
8 & LdBPK_080950 & 1.67 & 0.0981 & 0.1545 & Cathepsin L-Like Protease (Fragment) \\
15 & LdBPK_151140 & 1.66 & 0.0000 & 0.0000 & Tryparedoxin Peroxidase \\
\hline
\end{tabular}

to duplication may lead to an increase in the transcript levels of multicopy genes [43]. Among the genes with a significantly higher copy number in VL-SL compared to CL-SL were those involved in energy metabolism and amino acid metabolism. These potential functional differences may modulate immune responses by the host cells within which the parasites grow and survive, consequently contributing to distinct disease phenotypes. Striking differences in amino acid use and metabolism have been reported previously between Leishmania species associated with different phenotypes [50]. Several other genes with higher copy numbers in VL-SL, were those associated with virulent features favoring tropism for visceral organs. It is evident from these results that marked chromosome and gene copy number variations can be present within isolates of the same species, which may be adaptive virulence mechanisms.

The $A 2$ gene locus, which has been the focus of several studies exploring the parasite's invasive characteristics during infection $[14,51,52]$, was not seen to be differentially distributed among CL-SL and VL-SL groups in our study. This finding suggests that intra

Table 7 Tandem gene arrays with highly variable copy numbers between L. donovani CL-WT and CL-PR

\begin{tabular}{|c|c|c|c|c|c|}
\hline Chr & OrthoMCL ID & Product description & Haploid CL-WT & Haploid CL-PR & CL-PR/CL-WT ratio \\
\hline 32 & OG5_134607 & Hypothetical protein, conserved & 1 & 5 & 3.42 \\
\hline 34 & OG5_131023 & Quinonoid dihydropteridine reductase & 1 & 3 & 2.35 \\
\hline 34 & OG5_143904 & Amastin surface glycoprotein, putative & 3 & 5 & 1.80 \\
\hline 12 & OG5_145891 & Hypothetical protein, conserved & 1 & 2 & 1.70 \\
\hline 21 & OG5_136877 & Palmitoyl acyltransferase 3 , putative & 1 & 2 & 1.68 \\
\hline 32 & OG5_157991 & Hypothetical protein, conserved & 1 & 2 & 1.65 \\
\hline 32 & OG5_166761 & GIPL galf transferase, putative & 1 & 2 & 1.65 \\
\hline 36 & OG5_184184 & Hypothetical protein, conserved & 1 & 2 & 1.62 \\
\hline 35 & OG5_204252 & Hypothetical protein, conserved & 1 & 2 & 1.59 \\
\hline 4 & OG5_148241 & Hypothetical protein, conserved & 2 & 3 & 1.58 \\
\hline 36 & OG5_126983 & 40 S ribosomal protein 59 , putative & 1 & 2 & 1.57 \\
\hline 36 & OG5_158724 & Histidine secretory acid phosphatase, putative & 1 & 2 & 1.56 \\
\hline 35 & OG5_130002 & Yos1-like, putative & 1 & 2 & 1.51 \\
\hline 36 & OG5_127039 & Sugar efflux transporter for intercellular exchange, putative & 1 & 2 & 1.50 \\
\hline 35 & OG5_128319 & ADP-ribosylation factor-like 2, arl2, putative & 1 & 2 & 1.50 \\
\hline 27 & OG5_127093 & D-lactate dehydrogenase-like protein & 5 & 2 & 0.41 \\
\hline
\end{tabular}


species variations resulting in different tropisms are likely to be influenced by many genes and their cumulative effects, as compared to phenotypic differences being determined by variations in a single gene locus or region. Transcriptional data from multiple isolates would add to the functional relevance of above observations.

The SNP based phylogenetic analysis suggested genetic diversity of $L$. donovani in Sri Lanka to be much higher than previously shown [7]. According to the phylogenetic tree, CL-SL and VL-SL strains have clearly different genetic origins and they might have established themselves much earlier than the ones in India and Nepal. Notably, the L. infantum branch seemed shorter than expected. This might have been caused by the fact that the reads were mapped to a $L$. donovani reference, and where $L$. infantum is very different from $L$. donovani, the reads that could not be mapped to the regions remained as gaps, likely leading to a shorter branch length.

The SNP analysis showed CL-SL and VL-SL to form two distinct groups with the CL-PR group also clustering separately. Non-synonymous SNPs present in coding regions are deemed particularly important because they could lead to changes at the protein level. Such variations unique to isolates from one phenotype may indicate functional differences which influence its characteristics. However, the distribution of non-synonymous SNPs among our isolates did not show major differences that could possibly explain the phenotypic differences. A previous phylogenomic study of clinical isolates of $L$. donovani from the Indian subcontinent showed Sri Lankan isolates to be closely related to the core group (ISC2-10) of samples as described in its [7] analysis. None of the unique SNPs or indels which could be used to identify each of these populations [53], as suggested subsequently as an alternative to whole genome sequencing for epidemiological purposes, were present in our isolates.

Drug resistance in Leishmania has often been related to copy number variations in specific genes although specific RNA levels have also been detected to change without affecting gene copy number [54-56]. We observed several genes associated with resistance to antimonials as well as some genes that can be attributed to vital biological processes such as parasite growth and survival, to be present in higher copy numbers in the poor responders to treatment (CL-PR). Similar associations were also seen with some genes with high counts of non-synonymous SNPs among our CL-PR group. The presence of amplifications at H-Locus, which has been implicated in drug resistance $[47,57]$, was more ambiguous with this being observed in CL-WT as well as VL-SL. Poor response to treatment in leishmaniasis can be attributed to many causes including variability in drug quality, dose, technique of administration and host factors. Thus, demonstration of true drug resistance of parasites requires confirmation with in vitro drug sensitivity testing. However, variations at parasite gene/ genome level in the background of such clinical observations provide valuable information on possible candidates for further study.

\section{Conclusions}

This comparative analysis of whole genome sequences attempted to elucidate genetic factors of virulence in $L$. donovani clinical isolates, largely responsible for an unusual presentation of cutaneous leishmaniasis. A range of genomic differences were observed predominantly at chromosome and gene level and to some extent in SNP distribution in the CL and VL isolates. Our results further indicate that aneuploidy is common and the pattern of inter-strain aneuploidy and intra-strain mosaicism vary within the $\mathrm{CL}$ and VL isolates of Sri Lankan $L$. donovani, possibly giving rise to differential expression of genes, ultimately leading to different disease tropisms.

Overall, the genes implicated are mostly those involved in growth and metabolic processes suggesting that adaptive virulence mechanisms may play a significant role in the pathogenesis by the different isolates. While we recognize that the lower number of $\mathrm{VL}$ isolates is likely to have affected the statistical comparisons, increasing the number of samples should be complemented by analysis at transcriptional and functional level to validate these findings. It is also known that the sequencing technology as well as the sequencing kits used can affect the subsequent analyses.

To our knowledge, this is the first genomic analysis of $L$. donovani strains demonstrating poor response to treatment in a cutaneous phenotype. In a background where the mechanisms of action of the widely used drugs still remain obscure, especially within a clinical setting, this information adds to the possible parasite genetic determinants of response to antimonials. The occurrence of $\mathrm{H}$-locus amplification in our VL isolates which have not been exposed to antimonials highlights the need for further evaluation of these potential molecular markers.

The genomic differences identified in this study contribute to refining the understanding of molecular mechanisms underlying pathogenesis and drug responses of this parasite with known genome plasticity. While the findings favor the presence of different strains which may result in distinct phenotypes, host immune response determinants as well as sand fly interactions should be taken into consideration in interpreting the contribution of these genetic differences.

\section{Methods}

\section{Sample collection}

Six cutaneous leishmaniasis (CL-SL) and two visceral leishmaniasis (VL-SL) clinical isolates were obtained 
from Sri Lankan patients (Additional file 1: Table S1-(A)). All patients had no travel history out of the country and were residents of areas endemic for leishmaniasis.

The CL-SL clinical isolates were derived from aspirates or punch biopsies of skin lesions while the VL-SL clinical isolates were from bone marrow aspirates. Among the CL-SL isolates two were reported as poor responders (CL-PR) to SSG. Patients who did not demonstrate satisfactory healing after weekly intra-lesional SSG for 10 weeks were identified as poor responders in the local clinical setting, as per established criteria referred previously [58].

\section{Genomic DNA library preparation and sequencing}

Genomic DNA was extracted from CL-SL and VL-SL clinical isolates to prepare paired-end DNA sequencing libraries of $500 \mathrm{bp}$ fragments using Nextera DNA sequencing library preparation kits. $300 \mathrm{bp}$ paired-end reads (Additional file 1: Table S1-(B)) were generated from these DNA sequencing libraries on an Illumina MiSeq platform at the Human Genetics Unit, Faculty of Medicine, University of Colombo, Sri Lanka.

\section{Preliminary analysis}

The genome reference of Leishmania donovani BPK282A1 strain from Nepal obtained from GeneDB database (LdBPK282V1) [59] was used as the reference genome for the analysis. Sequencing reads of each CL-SL and VL-SL samples were analyzed with the use of FastQC tool [60] for base quality and overall GC content distribution. The paired-end reads from each sample were mapped to the $L$. donovani BPK282A1 reference using BWA-mem tool [61]. Mapped BAM files were cleaned, sorted, validated and duplicates were marked using Picard tool [62]. GATK toolkit [63] was used for further processing and analysis of the reads. Mapping and variant calling were repeated adhering to the same protocol against the recently generated reference for $L$. donovani on a Pacific Biosciences (PacBio) sequencing platform (LdBPK282V2) [30]. This new reference is more complete at repetitive regions but all public databases such as TriTrypDB [3] still maintain the previous version. We therefore performed the main analyses based on the genome reference LdBPK282V1.

\section{Chromosome and gene copy number analysis}

Chromosome copy numbers/somy were calculated for each chromosome in each sample separately as described elsewhere $[6,7,30]$. Read depth for each position of chromosome was obtained using SAMtools [64]. Median read depth of each chromosome was divided by median of all chromosomal medians of a sample to get a raw somy value for a chromosome. A custom written python script "Chromo_median.py" was used to automate these calculations. This raw somy value was adjusted using the 4 neighboring chromosomes on each side and the chromosome itself. Adjusted somy value for a chromosome was calculated by dividing the median somy of these 9 chromosomes by the raw somy value of the chromosome [30] (Additional file 1: Table S2). Somy (S) was identified as monosomy, disomy, trisomy, tetrasomy and pentasomy as previously described by others as $\mathrm{S}<1.5, \quad 1.5 \leq \mathrm{S}<2.5,2.5 \leq \mathrm{S}<3.5, \quad 3.5 \leq \mathrm{S}<4.5, \quad 4.5 \leq \mathrm{S}<$ 5.5 respectively $[6,7,30,43]$. Chromosome copy number data was saved in an 8 by 36 numeric matrix and similarity measures were calculated between the $8 \mathrm{~L}$. donovani isolates and their aneuploidy profiles by VCFtools. This data was then used to generate a heat map with heatmap.2() function.

Gene copy number was calculated for each gene from each library as described previously [14, 43]. Read depth for each position of each gene on a chromosome was obtained using SAMtools. A bed file containing L. donovani gene positions was used to achieve this. A custom written python script "Gene_median.py" was used to obtain median read coverage per gene, and it was divided by the median coverage of the entire chromosome to obtain a normalized copy number for the gene. These values were used to calculate an average read depth per gene for the CL-SL and VL-SL groups and genes with an average read depth per gene $>0.5$ were retained. A VL/ $\mathrm{CL}$ ratio per gene was calculated based on these average values and genes with at least a two-fold change in copy number (either direction) between the Sri Lankan VL and CL isolates, were assumed to have biologically significant implications to their functions, and were used for downstream analysis. This set of genes was also evaluated for statistically significant copy number changes by applying Mann-Whitney U (MWU) test [65] with Benjamini and Hochberg false discovery rate (FDR) correction [66] for multiple comparisons. As the small number of samples limits the robustness of statistical testing, we defined each read as an observation point and used normalized read depth values of all positions within a gene as the data set, to take advantage of genome sequencing read information for the statistical comparison. Multicopy genes/tandem arrays, which are genes of more than one copy with the same OrthoMCL group ID and present on the same chromosome, were identified using OrthoMCL-DB [67]. Read coverage across chromosomal regions were visualized using Artemis tool [68] and Integrative Genomics Viewer [69].

\section{SNP and Indel analysis}

SNPs and small indels were analyzed in detail in the three distinct phenotypes; CL-SL, VL-SL and CL-PR in comparison to the $L$. donovani reference. GATK's HaplotypeCaller (HC) module was used in ERC GVCF mode to call SNPs and indels in each sample using 
minimum base quality (-mbq) of 21 and -stand_call_ conf 30. HC produced .gvcf files were joined using GATK's GenotypeGVCFs. The resultant multi-sample vcf file with raw variants were filtered for SNPs and indels respectively. These SNPs and indels were hard-filtered in order to extract high quality ones. $\mathrm{QD}<2.0, \mathrm{FS}>60.0, \mathrm{MQ}<40$, MQRankSum $<-12.5$, ReadPosRankSum $<-8.0$, SOR $>2.5$ thresholds were used to filter SNPs and QD $<2.0$, FS $>100.0$, ReadPosRankSum < -10.0 and SOR $>5.0$ as thresholds used to filter indels. SNPs and indels with read-depth coverage $(\mathrm{DP})<10$ and DP $>200$ were excluded from analysis, as well as QUAL < 1500. Maximum DP was set 5-6 standard deviations from the mean coverage across all samples. Additionally, genotype fields were filtered for read-depth coverage $(\mathrm{DP})<8$ and genotype quality $(\mathrm{GQ})<20$. Filtered sites, genotypes and non-variants were all excluded from analysis. SNPs and indels in regions of first $3 \mathrm{~kb}$ and last $5 \mathrm{~kb}$ of each chromosome were excluded to remove potential low quality variants. Tantan tool [70] was used to remove variants in low complexity repeat regions.

In order to obtain a consistent set of variants for each group of CL-SL and VL-SL, and to minimize incorrect variant calling, we retained the variants present in at least half of the samples in each group. The resultant vcf files were used as input for SnpEff [71] to annotate the variants. Genes likely to be under strong selection were identified based on a ratio of non-synonymous SNP counts $\left(\mathrm{P}_{\mathrm{N}}\right)$ to sum of synonymous $\left(\mathrm{P}_{\mathrm{S}}\right.$ and non-synonymous $\left(\mathrm{P}_{\mathrm{N}}\right)$ SNP counts above 0.5 .

VCFtools [72] and a multi-sample vcf file were used to calculate a relatedness measure among the $8 \mathrm{~L}$. donovani isolates based on SNP data and a similarity matrix was built up using R [73]. This matrix was used to generate a heat map using heatmap.2() function in gplots $\mathrm{R}$ package [74]. Bioconductor package SNPRelate [75] was used to remove SNPs in high linkage disequilibrium within our multi-sample vcf and to generate a pruned SNP set. This pruned SNP set was then used to carryout principal component analysis.

SNP allele heat maps were also created using the multi-sample vcf file with all $8 \mathrm{~L}$. donovani isolates. Perl scripts were used to extract only the allele frequency from the vcf per each chromosome and finally a SNP allele frequency file per each chromosome was obtained. Allele frequency per bin $(5 \mathrm{~kb})$ was calculated for each chromosome using a perl script and heat maps per chromosome were generated using heatmap.2() function. Finally a single heat map was generated combining all files per chromosome.

\section{Phylogenetic analysis}

We used a stringently filtered multi-sample vcf file consisting of our 8 Sri Lankan isolates and other key
Leishmania strains discussed previously [7], to describe the genetic divergence levels among them by maximum-likelihood (ML) based phylogenetic inference. A fasta alignment file was generated from the multi-sample vcf file. RAxML [76] was used to find the best scoring maximum likelihood tree for the alignment using bootstrap convergence criteria with rapid bootstrapping and selecting the GTRGAMMA as the model of substitution. Visualizing and further analysis of ML tree was done by FigTree [77].

\section{Gene ontology}

Gene ontology terms (GO terms) enriched within the set of differentially distributed genes among the two groups of CL-SL and VL-SL were identified by extracting the Leishmania major Friedlin (LmjF) orthologs corresponding to $L$. donovani genes and performing the GO enrichment analysis using TriTrypDB [3]. GO terms significantly overrepresented $(p$-value $<0.05)$ in the gene set for biological processes, molecular functions and cellular components were identified with Benjamini and Hochberg false discovery rate correction. Removal of the redundant GO terms in the set and generating scatterplots for the remaining terms were done using REVIGO, a web based semantic cluster algorithm [78].

\section{Additional files}

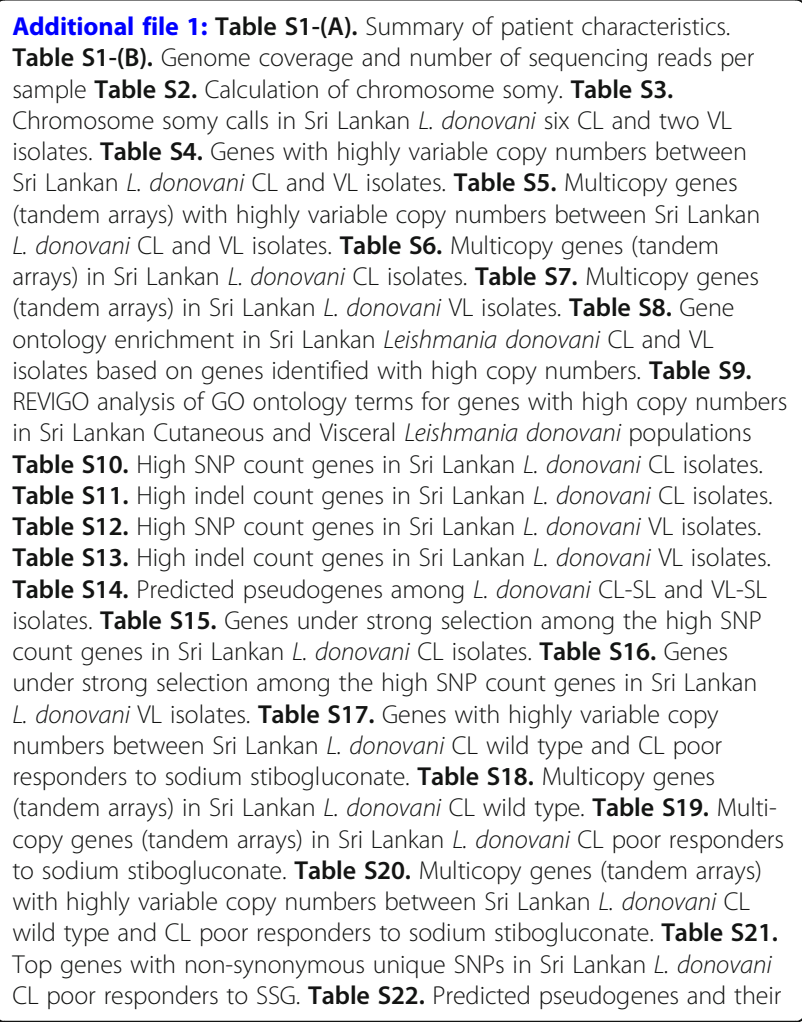


functions in $L$. donovani $C L$ wild type and $C L$ poor responders to sodium stibogluconate. (XLSX $961 \mathrm{~kb}$ )

Additional file 2: Figure S1. Read coverage (based on all the positions) across each chromosome of Sri Lankan L. donovani CL isolate L2339. (PDF $1585 \mathrm{~kb}$ )

Additional file 3: Figure S2. Read coverage (based on all the positions) across each chromosome of Sri Lankan L. donovani VL isolate VL38. (PDF $1587 \mathrm{~kb}$ )

Additional file 4: Figure S3. Comparison of read coverage across the A2 region in chromosome 22 of six CL-SL and two VL-SL clinical isolates. (PDF $62 \mathrm{~kb}$ )

Additional file 5: Figure S4. Two frameshift mutations on trypanothione reductase gene (LdBPK_050350) of Sri Lankan L. donovani. These two frameshifts are present in all the CL-SL isolates except R5_S5 and VL-SL isolates have intact copies. Frameshift at position Ld05_V01s1:104041 (left) is an insertion changing the reference ' $C$ ' allele to a ' $C T G$ '. The other frameshift is a deletion at position Ld05_v01s1:104042 (right) and it changes the reference 'CAG' allele in to a ' $C$ '. Both frameshifts are covered by more than $65 \%$ of the reads at both positions in all the $5 \mathrm{CL}-\mathrm{SL}$ isolates. Details were viewed in IGV. (PNG $23 \mathrm{~kb}$ )

Additional file 6: Figure S5. Heat map showing the SNP allele frequency distribution across chromosomes of Sri Lankan L. donovani isolates. Allele frequencies for $5 \mathrm{~kb}$ bins of all chromosomes from 1 to 36 from the top are on the $y$-axis and $L$. donovani $C L$ and $V L$ isolates are listed along the bottom $x$-axis. The colour bar represents the phenotype of isolate. Red-CL-SL and Black-VL-SL. The colour key with histogram represents the allele frequency distribution. (PNG $77 \mathrm{~kb}$ )

\section{Abbreviations}

CDS: Coding DNA sequence; CL: Cutaneous leishmaniasis; CL-PR: Cutaneous leishmaniasis poor responders to SSG; CL-SL: Cutaneous leishmniasis Sri Lanka; CL-WT: Cutaneous leishmaniasis wild type; GO: Gene ontology; LdAQP1: Leishmania donovani aquaglyceroporin 1; LmjF: Leishmania major Friedlin; MAPKs: Mitogen-activated protein kinases; MWU: Mann-Whitny U test; S: Chromosome somy; SNP: Single nucleotide polymorphism; SSG: Sodium stibogluconate; VL: Visceral leishmaniasis; VL-SL: Visceral leishmaniasis Sri Lanka

\section{Acknowledgements}

We would like to thank Prof. V.H.W. Dissanayake of the Human Genetics Unit, Faculty of Medicine, University of Colombo, Sri Lanka for provision of sequencing facilities.

\section{Funding}

Research reported in this publication was supported by the National Institute of Allergy and Infectious Diseases of the National Institutes of Health under Award Numbers U01Al136033 and R01Al099602. The content is solely the responsibility of the authors and does not necessarily represent the official views of the National Institutes of Health.

\section{Availability of data and materials}

Most of the data supporting the conclusions of this article are included within the article and its Additional files. This project has been submitted to GenBank under bioproject ID PRJNA413320. The genome datasets of the eight $L$. donovani isolates have been deposited in the NCBI Sequence Read Archive (SRA) repository, under accession number SRP124074. Other datasets used and/or analyzed during the current study are available from the corresponding author on reasonable request.

\section{Authors' contributions}

NDK and NS designed the study and conceptualized the methodology. ULK and YDS collected and maintained the data and samples, recovered and provided the clinical isolates and related information. NS performed the laboratory procedures related to sequencing with assistance from ULK and SRS. SRS performed the bioinformatics analysis and wrote the manuscript $\mathrm{HI}$ analyzed and interpreted data and revised the manuscript critically. NS and NDK revised the manuscript critically and supervised the study. All authors read and approved the final manuscript.

\section{Ethics approval and consent to participate}

This study was conducted according to a protocol approved by the Ethics Review Committee of Faculty of Medicine, University of Colombo (EC/12/ 068), Sri Lanka. Written informed consent was obtained from the patients prior to data and sample collection.

\section{Consent for publication}

Not applicable.

\section{Competing interests}

The authors declare that they have no competing interests.

\section{Publisher's Note}

Springer Nature remains neutral with regard to jurisdictional claims in published maps and institutional affiliations.

\section{Author details}

${ }^{1}$ Department of Parasitology, Faculty of Medicine, University of Colombo, Colombo, Sri Lanka. ${ }^{2}$ Department of Biomedical Sciences, Institute of Tropical Medicine, Antwerp, Belgium.

Received: 5 January 2018 Accepted: 19 November 2018 Published online: 28 November 2018

\section{References}

1. Alvar J, Vélez ID, Bern C, Herrero M, Desjeux P, Cano J, et al. Leishmaniasis worldwide and global estimates of its incidence. PLoS One. 2012. https://doi.org/10.1371/journal.pone.0035671.

2. Cantacessi C, Dantas-Torres F, Nolan MJ, Otranto D. The past, present, and future of Leishmania genomics and transcriptomics. Trends Parasitol. 2015;31:100-8.

3. Aslett M, Aurrecoechea C, Berriman M, Brestelli J, Brunk BP, Carrington M, et al. TriTrypDB: a functional genomic resource for the Trypanosomatidae. Nucleic Acids Res. 2009;38:457-62.

4. Peacock CS, Seeger K, Harris D, Murphy L, Ruiz JC, Quail MA, et al. Comparative genomic analysis of three Leishmania species that cause diverse human disease. Nat. Genet. 2007:39:839-47.

5. Kumar S, Gokulasuriyan RK, Ghosh M. Comparative in-silico genome analysis of Leishmania (Leishmania) donovani: a step towards its species specificity. Meta Gene. 2014;2:782-98.

6. Downing T, Imamura H, Decuypere S, Clark TG, Coombs GH, Cotton JA, et al. Whole genome sequencing of multiple Leishmania donovani clinical isolates provides insights into population structure and mechanisms of drug resistance. Genome Res. 2011:21:2143-56.

7. Imamura H, Downing T, Van den Broeck F, Sanders MJ, Rijal S, Sundar S, et al. Evolutionary genomics of epidemic visceral leishmaniasis in the Indian subcontinent. elife. 2016. https://doi.org/10.7554/eLife.12613.

8. Karunaweera ND, Pratlong F, Siriwardane HVYD, Ihalamulla RL, Dedet JP. Sri Lankan cutaneous leishmaniasis is caused by Leishmania donovani zymodeme MON-37. Trans R Soc Trop Med Hyg. 2003. https://doi.org/10. 1016/s0035-9203(03)90061-7.

9. Rajapaksa US, Ihalamulla RL, Karunaweera ND. First report of mucosal tissue localisation of leishmaniasis in Sri Lanka. Ceylon Med J. 2005;50:90-1.

10. Karunaweera ND. Leishmania donovani causing cutaneous leishmaniasis in Sri Lanka: a wolf in sheep's clothing? Trends Parasitol. 2009;25:458-63.

11. Refai FW, Madarasingha NP, Fernandopulle R, Karunaweera N. Nonresponsiveness to standard treatment in cutaneous leishmaniasis: a case series from Sri Lanka. Trop Parasitol. 2016;6:155-8.

12. Siriwardana HVYD, Noyes HA, Beeching NJ, Chance ML, Karunaweera ND, Bates PA. Leishmania donovani and Cutaneous Leishmaniasis, Sri Lanka. Emerg Infect Dis. 2007;13:1-3.

13. Kariyawasam UL, Selvapandiyan A, Rai K, Wani TH, Pahuja K, Premathilake $\mathrm{HU}$, et al. Genetic diversity of Leishmania donovani that causes cutaneous leishmaniasis in Sri Lanka: a cross sectional study with regional comparisons. BMC Infect Dis. 2017. https://doi.org/10.1186/s12879-017-2883-X.

14. Zhang WW, Ramasamy G, Mccall L, Haydock A. Genetic analysis of Leishmania donovani tropism using a naturally attenuated cutaneous strain. PLoS Pathog. 2014. https://doi.org/10.1371/journal.ppat.1004244.

15. Myler PJ, Fasel N, editors. The metabolic repertoire of Leishmania and implications for drug discovery. Leishmania: After the Genome. Caister Academic Press; 2008. p. 133-4. 
16. Ariza A, Vickers TJ, Greig N, Armour KA, Dixon MJ, Eggleston IM, et al. Specificity of the trypanothione-dependent Leishmania major glyoxalase : structure and biochemical comparison with the human enzyme. Mol Microbiol. 2006:59:1239-48.

17. Olivier M, Atayde VD, Isnard A, Hassani K, Shio MT. Leishmania virulence factors: focus on the metalloprotease GP63. Microbes Infect. 2012;14:1377-89.

18. Seay MB, Heard PL, Chaudhuri G. Surface Zn-proteinase as a molecule for defense of Leishmania mexicana amazonensis promastigotes against cytolysis inside macrophage phagolysosomes. Infect Immun. 1996;64:5129-37.

19. Nunes VS, Damasceno JD, Freire R, Tosi LRO. Molecular \& biochemical parasitology the Hus1 homologue of Leishmania major encodes a nuclear protein that participates in DNA damage response. Mol Biochem Parasitol. 2011;177:65-9.

20. Vickers TJ, Beverley SM. Folate metabolic pathways in Leishmania. Essays Biochem. 2011;51:63-80.

21. Nare B, Hardy LW, Beverley SM, Ptrs L. The roles of Pteridine reductase 1 and Dihydrofolate reductase-thymidylate synthase in Pteridine metabolism in the protozoan parasite Leishmania major *. J Biol Chem. 1997;272:13883-91.

22. Cunningham ML, Beverley SM. Pteridine salvage throughout the Leishmania infectious cycle: implications for antifolate chemotherapy. Mol Biochem Parasitol. 2001;113:199-213.

23. Baharia RK, Tandon R, Sahasrabuddhe AA, Sundar S, Dube A. Nucleosomal histone proteins of $L$. donovani: a combination of recombinant $\mathrm{H} 2 \mathrm{~A}, \mathrm{H} 2 \mathrm{~B}, \mathrm{H} 3$ and $\mathrm{H} 4$ proteins were highly immunogenic and offered optimum prophylactic efficacy against Leishmania challenge in hamsters. PLoS One. 2014;9(6):e97911.

24. Gowri V, Ghosh I, Sharma A, Madhubala R. Unusual domain architecture of aminoacyl tRNA synthetases and their paralogs from Leishmania major. BMC Genomics. 2012. https://doi.org/10.1186/1471-2164-13-621.

25. Hashimoto M, Murata E, Aoki T. Secretory protein with RING finger domain (SPRING) specific to Trypanosoma cruzi is directed, as a ubiquitin ligase related protein, to the nucleus of host cells. Cell Microbiol. 2009:12:19-30.

26. Zhang WW, Matlashewski G. Characterization of the A2-A2rel gene cluster in Leishmania donovani: involvement of A2 in visceralization during infection. Mol Microbiol. 2001;39:935-48.

27. Jackson AP. The evolution of amastin surface glycoproteins in trypanosomatid parasites. Mol Biol Evol. 2010;27:33-45.

28. Tovar J, Wilkinson S, Mottram JC, Fairlamb AH. Evidence that trypanothione reductase is an essential enzyme in Leishmania by targeted replacement of the tryA gene locus. Mol Microbiol. 1998;29:653-60.

29. Junghae M, Raynes JG. Activation of p38 mitogen-activated protein kinase attenuates Leishmania donovani infection in macrophages activation of p38 mitogen-activated protein kinase attenuates Leishmania donovani infection in macrophages. Infect Immun. 2002;70:5026-35.

30. Dumetz F, Imamura H, Sanders M, Seblova V, Myskova J, Pescher P. Modulation of aneuploidy in Leishmania donovani during adaptation to different in vitro and in vivo environments and its impact on gene expression. MBio. 2017. https://doi.org/10.1128/mBio.00599-17.

31. Fuente SG, Peiró-p R, Rastrojo A, Moreno J, Carrasco-ramiro F, Requena JM et al. Resequencing of the Leishmania infantum ( strain JPCM5) genome and de novo assembly into 36 contigs. Sci Rep. 2017;7:1-10.

32. Cotrim PC, Garrity LK, Beverley SM. Isolation of genes mediating resistance to inhibitors of nucleoside and Ergosterol metabolism in Leishmania by overexpression/selection. J Biol Chem. 1999;274:37723-30.

33. El Fadili K, Drummelsmith J, Roy G, Jardim A, Ouellette M. Down regulation of KMP-11 in Leishmania infantum axenic antimony resistant amastigotes as revealed by a proteomic screen. Exp. Parasitol. 2009;123:51-7.

34. Sardar AH, Jardim A, Ghosh AK, Mandal A, Das S, Saini S, et al. Genetic manipulation of Leishmania donovani to explore the involvement of Argininosuccinate synthase in oxidative stress management. PLoS Negl Trop Dis. 2016;10:e0004308.

35. Lakhal-Naouar I, Jardim A, Strasser R, Luo S, Kozakai Y, Nakhasi HL, et al. Leishmania donovani Argininosuccinate synthase is an active enzyme associated with parasite pathogenesis. PLoS Negl Trop Dis. 2012;6:e1849

36. Iyer JP, Kaprakkaden A, Choudhary ML, Shaha C. Crucial role of cytosolic tryparedoxin peroxidase in Leishmania donovani survival, drug response and virulence. Mol Microbiol. 2008;68:372-91.

37. JA S, SA N, VJ C, JC E, Leptak C, Bouvier J. Leishmania major: comparison of the Cathepsin L- and B-like cysteine protease genes with those of other Trypanosomatids. Exp Parasitol. 1997;85:63-76

38. Somanna A, Mundodi V, Gedamu L. Functional analysis of Cathepsin B-like cysteine proteases from Leishmania donovani complex. J Biol Chem. 2002;277:25305-12.
39. Lye LF, Cunningham ML, Beverley SM. Characterization of quinonoiddihydropteridine reductase (QDPR) from the lower eukaryote Leishmania major. J Biol Chem. 2002;277:38245-53.

40. Wu Y, El Fakhry Y, Sereno D, Tamar S, Papadopoulou B. A new developmentally regulated gene family in Leishmania amastigotes encoding a homolog of amastin surface proteins. Mol Biochem Parasitol. 2000;110:345-57.

41. Adung'a VO, Gadelha C, Field MC. Proteomic analysis of Clathrin interactions in trypanosomes reveals dynamic evolution of endocytosis. Traffic. 2013;14: 440-57.

42. Ashutosh GM, Sundar S, Duncan R, Nakhasi HL, Goyal N. Downregulation of mitogen-activated protein kinase 1 of Leishmania donovani field isolates is associated with antimony resistance. Antimicrob Agents Chemother. 2012;56:518-25.

43. Rogers MB, Hilley JD, Dickens NJ, Wilkes J, P a B, Depledge DP, et al. Chromosome and gene copy number variation allow major structural change between species and strains of Leishmania. Genome Res. 2011;21:2129-42.

44. Valdivia HO, Reis-cunha JL, Rodrigues-luiz GF, Baptista RP, Baldeviano GC, Gerbasi RV, et al. Comparative genomic analysis of Leishmania ( Viannia ) peruviana and Leishmania ( Viannia ) braziliensis. BMC Genomics. 2015. https://doi.org/10.1186/s12864-015-1928-z.

45. Sterkers Y, Lachaud L, Bourgeois N, Crobu L, Bastien P, Pagès M. Novel insights into genome plasticity in eukaryotes : mosaic aneuploidy in Leishmania. Mol Microbiol. 2012;86:15-23.

46. Depledge DP, Evans KJ, Ivens AC, Aziz N, Maroof A, Kaye PM, et al. Comparative expression profiling of Leishmania: modulation in gene expression between species and in different host genetic backgrounds. PLoS Negl Trop Dis. 2009;3:7.

47. Leprohon P, Légaré D, Raymond F, Madore É, Hardiman G, Corbeil J, et al. Gene expression modulation is associated with gene amplification, supernumerary chromosomes and chromosome loss in antimony-resistant Leishmania infantum. Nucleic Acids Res. 2009;37:1387-99.

48. Mukherjee A, Langston LD, Ouellette M. Intrachromosomal tandem duplication and repeat expansion during attempts to inactivate the subtelomeric essential gene GSH1 in Leishmania. Nucleic Acids Res. 2011;39:7499-511.

49. Mukheriee A, Boisvert S, Monte-neto RL, Coelho AC, Raymond F, Mukhopadhyay R, et al. Telomeric gene deletion and intrachromosomal amplification in antimonyresistant Leishmania. Mol Microbiol. 2013;88:189-202.

50. Westrop GD, Williams RAM, Wang L, Zhang T, Watson DG, Silva AM, et al. Metabolomic analyses of Leishmania reveal multiple species differences and large differences in amino acid metabolism. PLoS One. 2015;10:9.

51. Fernandes AP, Canavaci AMC, McCall L-I, Matlashewski G. A2 and other Visceralizing proteins of Leishmania: role in pathogenesis and application for vaccine development. Subcell Biochem. 2014;74:77-101.

52. Zhang WW, Mendez S, Ghosh A, Myler P, Ivens A, Clos J, et al. Comparison of the A2 gene locus in Leishmania donovani and Leishmania major and its control over cutaneous infection. J Biol Chem. 2003;278:35508-15.

53. Rai K, Bhattarai NR, Vanaerschot M, Imamura H, Gebru G, Khanal B, et al. Single locus genotyping to track Leishmania donovani in the Indian subcontinent: application in Nepal. PLoS Negl Trop Dis. 2017;11:1-13.

54. Haimeur A, Guimond C, Pilote S, Mukhopadhyay R, Rosen BP, Poulin R, et al. Elevated levels of polyamines and trypanothione resulting from overexpression of the ornithine decarboxylase gene in arsenite-resistant Leishmania. Mol Microbiol. 1999;34:726-35.

55. Guimond C, Trudel N, Brochu C, Marquis N, El Fadili A, Peytavi R, et al. Modulation of gene expression in Leishmania drug resistant mutants as determined by targeted DNA microarrays. Nucleic Acids Res. 2003;31:5886-96.

56. Marquis N, Gourbal B, Rosen BP, Mukhopadhyay R, Ouellette M. Modulation in aquaglyceroporin AQP1 gene transcript levels in drug-resistant Leishmania. Mol Microbiol. 2005;57:1690-9.

57. El Fadili K, Messier N, Leprohon P, Roy G, Guimond C, Trudel N, et al. Role of the $A B C$ transporter MRPA (PGPA) in antimony resistance in Leishmania infantum axenic and intracellular amastigotes. Antimicrob Agents Chemother. 2005;49:1988-93.

58. Refai WF, Madarasingha NP, Sumanasena B, Weerasingha S, De Silva A, Fernandopulle $R$, et al. Efficacy, safety and cost-effectiveness of thermotherapy in the treatment of Leishmania donovani-induced cutaneous Leishmaniasis: a randomized controlled clinical trial. Am J Trop Med Hyg. 2017;97:1120-6. 
59. Logan-Klumpler FJ, De Silva N, Boehme U, Rogers MB, Velarde G, McQuillan JA, et al. GeneDB — an annotation database for pathogens. Nucleic Acids Res. 2012:40:D98-D108.

60. Andrews S. FastQC: a quality control tool for high throughput sequence data. http://www.bioinformatics.babraham.ac.uk/projects/fastqc. Accessed 1 Sept 2016.

61. Li H. Aligning sequence reads, clone sequences and assembly contigs with BWA-MEM. 2013;ArXiv:1303.3997 [q-bio.GN].

62. Broad Institute. Picard. http://broadinstitute.github.io/picard. Accessed 1 Sept 2016.

63. McKenna A, Hanna M, Banks E, Sivachenko A, Cibulskis K, Kernytsky A, et al. The genome analysis toolkit: a MapReduce framework for analyzing next-generation DNA sequencing data. Genome Res. 2010;20:1297-303.

64. Li H, Handsaker B, Wysoker A, Fennell T, Ruan J, Homer N, et al. The sequence alignment/map format and SAMtools. Bioinformatics. 2009;25:2078-9

65. Mann HB, Whitney DR. On a test of whether one of two random variables is stochastically larger than the other. Ann Math Stat. 1947;18:50-60.

66. Benjamini $Y$, Hochberg $Y$. Controlling the false discovery rate: a practical and powerful approach to multiple testing. J R Stat Soc Ser B. 1995;57:289-300,

67. Chen F, Mackey AJ, Christian J, Stoeckert J, Roos DS. OrthoMCL-DB: querying a comprehensive multi-species collection of ortholog groups. Nucleic Acids Res. 2006;34:D363-8.

68. Rutherford K, Parkhill J, Crook J, Horsnell T, Rice P. Artemis : sequence visualization and annotation. Bioinforma Appl Note. 2000;16:944-5.

69. Robinson JT, Thorvaldsdóttir $H$, Winckler W, Guttman M, Lander ES, Getz G, et al. Integrative genomics viewer. Nat Biotechnol. 2011;29:24-6.

70. Frith MC. A new repeat-masking method enables specific detection of homologous sequences. Nucleic Acids Res. 2011. https://doi.org/10.1093/ $\mathrm{nar} / \mathrm{gkq} 1212$.

71. Cingolani P, Platts A, Wang LL, Coon M, Nguyen T, Wang L, et al. A program for annotating and predicting the effects of single nucleotide polymorphisms, SnpEff. Fly. 2012;6:80-92.

72. Danecek P, Auton A, Abecasis G, Albers CA, Banks E, DePristo MA, et al. The variant call format and VCFtools. Oxford Acad Bioinforma. 2011:27: 2156-8.

73. Team RCR. A language and environment for statistical computing In: R Foundation for statistical computing; 2014. http://www.R-project.org. Accessed 1 Oct 2017.

74. Warnes GR, Bolker B, Bonebakker L, Gentleman R, Liaw WHA, Lumley T, et al. Gplots: various R programming tools for plotting data. 2016. https://cran.rproject.org/package=gplots. Accessed 1 Oct 2017.

75. Zheng X, Levine D, Shen J, Gogarten S, Laurie C, Weir B. A high-performance computing toolset for relatedness and principal component analysis of SNP data. Bioinformatics. 2012;28:3326-8.

76. Stamatakis A. RAxML version 8: a tool for phylogenetic analysis and postanalysis of large phylogenies. Bioinformatics. 2014;30:1312-3.

77. Rambaut A. FigTree: molecular evolution, Phylogenetics and epidemiology http://tree.bio.ed.ac.uk/software/figtree/. Accessed 1 Apr 2018.

78. Supek F, Bošnjak M, Škunca N, Šmuc T. REVIGO summarizes and visualizes long lists of gene ontology terms. PLoS One. 2011;6:e21800.

Ready to submit your research? Choose BMC and benefit from:

- fast, convenient online submission

- thorough peer review by experienced researchers in your field

- rapid publication on acceptance

- support for research data, including large and complex data types

- gold Open Access which fosters wider collaboration and increased citations

- maximum visibility for your research: over $100 \mathrm{M}$ website views per year

At BMC, research is always in progress.

Learn more biomedcentral.com/submissions 